\title{
Decay of Hamiltonian Breathers Under Dissipation
}

\author{
Jean-Pierre Eckmann ${ }^{1}$, C. Eugene Wayne ${ }^{2}$ (] \\ 1 Département de Physique Théorique and Section de Mathématiques, Université de Genève, \\ 1211 Geneva 4, Switzerland. \\ 2 Department of Mathematics and Statistics, Boston University, Boston, MA 02215, USA. \\ E-mail: cew@bu.edu
}

\begin{abstract}
We study metastable behavior in a discrete nonlinear Schrödinger equation from the viewpoint of Hamiltonian systems theory. When there are $n<\infty$ sites in this equation, we consider initial conditions in which almost all the energy is concentrated in one end of the system. We are interested in understanding how energy flows through the system, so we add a dissipation of size $\gamma$ at the opposite end of the chain, and we show that the energy decreases extremely slowly. Furthermore, the motion is localized in the phase space near a family of breather solutions for the undamped system. We give rigorous, asymptotic estimates for the rate of evolution along the family of breathers and the width of the neighborhood within which the trajectory is confined.
\end{abstract}

\section{Contents}

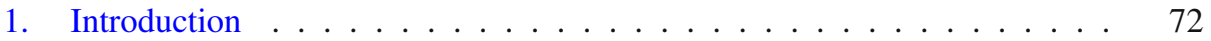

2. The Eigenspace of the Eigenvalue $0 \ldots \ldots$. . . . . . . . . . . . . . . . . 76

3. Evolution Equations for $\gamma>0 \ldots \ldots$. . . . . . . . . . . . . . . . . . . . . . . .

4. Spectral Properties of the Linearization at $\gamma=0$. . . . . . . . . . . . . 80

5. Complement of the Zero Eigenspace . . . . . . . . . . . . . . . . 83

6. The Effect of the Dissipation on the Semigroup . . . . . . . . . . . . . . 87

7. Projecting onto the Complement of the 0 Eigenspace . . . . . . . . . . 88

8. Bounds on the Evolution of $\zeta \ldots \ldots$. . . . . . . . . . . . . . . . . . . . . . . .

9. Re-orthogonalization . . . . . . . . . . . . . . . . . . . . . . . 97

9.1 The intuitive picture . . . . . . . . . . . . . . . . . . . 98

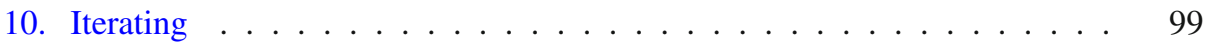

11. Conclusions and Future Directions . . . . . . . . . . . . . . . . 101 


\section{Introduction}

In the present work we look at the problem of a finite, discrete nonlinear Schrödinger equation, with dissipation, which we considered first in [1]. We need to repeat several equations from that paper, but the aim is now to give a complete proof of the observations and assertions in that paper. One starts with

$$
-\mathbf{i} \frac{\partial u_{j}}{\partial \tau}=-(\Delta u)_{j}+\left|u_{j}\right|^{2} u_{j}, j=1,2, \ldots, n,
$$

where we will add dissipation later. Here $(\Delta u)_{j}=u_{j-1}-2 u_{j}+u_{j+1}$, with free end boundary conditions for $j=1$ or $n$, i.e., $(\Delta u)_{1}=-u_{1}+u_{2}$ and $(\Delta u)_{n}=-u_{n}+u_{n-1}$.

For the convenience of the reader, the following introduction repeats the setup from [1].

We will choose initial conditions for this system in which essentially all of the energy is in mode $u_{1}$, and will add a weak dissipative term to the last mode as in [1,2] by adding to Eq. (1.1) a term of the form

$$
\mathbf{i} \gamma \delta_{n, j} u_{j},
$$

i.e., we add dissipation to position $n$, at the opposite end from the energetic mode.

Eventually, this will lead to the energy of the system tending to zero, but we are interested in what happens on intermediate time scales, and in particular, how the energy is transported from one end of the lattice to the other.

If our initial conditions are chosen so that $u_{1}(0)=\sqrt{\Omega}$, and all other $u_{j}(0)=0$, then we expect that at least initially, the coupling terms between the various modes will play only a small role in the evolution and the system will be largely dominated by the equation for $u_{1}$ :

$$
-\mathbf{i} \frac{\mathrm{d} u_{1}}{\mathrm{~d} \tau}=\left|u_{1}\right|^{2} u_{1},
$$

with solution $u_{1}(\tau)=\sqrt{\Omega} e^{\mathbf{i} \Omega \tau}$-i.e., we have a very fast rotation with large amplitude. With this in mind, we introduce a rescaled dependent variable and rewrite the equation in a rotating coordinate frame by setting:

$$
u_{j}(\tau)=\sqrt{\Omega} e^{\mathbf{i} \Omega \tau} \widetilde{w}_{j}(\tau)
$$

Then $\widetilde{w}_{j}$ satisfies

$$
\Omega \widetilde{w}_{j}-\mathbf{i} \frac{\partial \widetilde{w}_{j}}{\partial \tau}=-(\Delta \widetilde{w})_{j}+\Omega\left|\widetilde{w}_{j}\right|^{2} \widetilde{w}_{j}
$$

We now add dissipation by adding a term which acts on the last variable, with $\gamma \geq 0$,

$$
\Omega \widetilde{w}_{j}-\mathbf{i} \frac{\partial \widetilde{w}_{j}}{\partial \tau}=-(\Delta \widetilde{w})_{j}+\Omega\left|\widetilde{w}_{j}\right|^{2} \widetilde{w}_{j}+\mathbf{i} \gamma \delta_{n, j} \widetilde{w}_{j}
$$

Rearranging, and dividing by $\Omega$ gives

$$
-\mathbf{i} \frac{1}{\Omega} \frac{\partial \widetilde{w}_{j}}{\partial \tau}=-\frac{1}{\Omega}(\Delta \widetilde{w})_{j}-\widetilde{w}_{j}+\left|\widetilde{w}_{j}\right|^{2} \widetilde{w}_{j}+\mathbf{i} \frac{\gamma}{\Omega} \delta_{n, j} \widetilde{w}_{j} .
$$

Finally, we define $\varepsilon=\Omega^{-1}$, and rescale time so that $\tau=\varepsilon t$. Setting $w(t)=\widetilde{w}(\tau)$, we arrive finally at

$$
-\mathbf{i} \frac{\partial w_{j}}{\partial t}=-\varepsilon(\Delta w)_{j}-w_{j}+\left|w_{j}\right|^{2} w_{j}+\mathbf{i} \gamma \varepsilon \delta_{n, j} w_{j}
$$


Now that we have defined the main equation Eq. (1.3) we describe the picture that will be proved later:

When $\gamma=0$ and $|\varepsilon| \neq 0$ is small, this system possesses a family of breathers, namely solutions which in this rotating coordinate systems are stationary states in which most of the energy is localized in site $1 .{ }^{1}$ Such solutions take the form

$$
u_{\varepsilon, j}^{(0)} \sim(-1)^{j+1} \varepsilon^{j-1}, \quad j=1, \ldots, n .
$$

In fact, there are many such solutions, with different frequencies, which we will write as

$$
u_{\varepsilon, j}^{\left(\varphi_{0}\right)}=e^{\mathbf{i} \varphi_{0} t} p\left(\varphi_{0}\right)_{j},
$$

for $\varphi_{0}$ near zero, and $p\left(\varphi_{0}\right)_{j} \sim u_{\varepsilon, j}^{(0)}$. We will demonstrate the existence of these families of solutions in Theorem 1.6, using the implicit function theorem, and also give more accurate asymptotic formulas for them. As Eq. (1.3) is invariant under complex rotations, we actually have a circle of fixed points, with a phase we call $\vartheta$. As there is one such circle for every small $\varphi_{0}$ we represent, in Fig. 1, these solutions as a (green) cylinder, with the direction along the cylinder corresponding to changing $\varphi_{0}$ and motions "around" the cylinder corresponding to changing $\vartheta$.

When the dissipation is nonzero (i.e., when $\gamma>0$ ) these periodic solutions are destroyed, but they give rise to a family of time-dependent solutions which "wind" along the cylinder, the red curves in Fig. 1. We will prove that one can accurately approximate solutions of the dissipative equations by "modulating" the frequency and phase of the breather, namely we prove that the solutions of the dissipative equation can be written as:

$$
u_{j}(x, t)=e^{\mathbf{i}(t \varphi(t)+\vartheta(t))} u_{\varepsilon, j}^{(0)}+z_{j}(t), \quad j=1, \ldots, n,
$$

where

$$
\begin{aligned}
\dot{\varphi}(t) & \sim-2 \gamma \varepsilon^{2 n-1}, \\
t \dot{\varphi}(t)+\dot{\vartheta}(t) & \sim 0, \\
\|z(t)\| & \text { remains bounded by } \mathcal{O}\left(\gamma \varepsilon^{n}\right) .
\end{aligned}
$$

The higher order terms that we have omitted from these expressions are explicitly estimated in Sect. 7 .

We prove that the initial values $\varphi_{0}$ and $\vartheta_{0}$ can be chosen so that $z(t)$ is normal to the cylinder of breathers at the point $\left(\varphi_{0}, \vartheta_{0}\right)$, and that its long term boundedness is due to the (somewhat surprising) fact that the linearized dynamics about the family of breathers is uniformly (albeit weakly) damping in these normal directions. This is the main new technical result of the paper and the proof of this fact takes up Sects. 3-6. That breathers can play an important role in the non-equilibrium evolution of systems of coupled oscillators has also been discussed (non-rigorously) in the physics literature. For two recent examples see [5,6].

To formulate our results more precisely, we need some notation: Let $\delta(t) \equiv \varphi(t)-\varphi_{0}$. Let $s(t)=\int_{0}^{t} \mathrm{~d} \tau(\tau \dot{\varphi}(\tau)+\dot{\vartheta}(\tau))$. Let the initial condition be $\varphi_{0}, \vartheta_{0}$ and $z_{0}$, with $z_{0}$ perpendicular to the tangent space to the cylinder at $\varphi_{0}, \vartheta_{0}$. Note that $\delta(0)=s(0)=0$.

1 The existence and properties of breather solutions in infinite lattices of oscillators are discussed in [3] or [4]. The proofs in those cases are easily modified (and actually somewhat simpler) in the case of finitely many degrees of freedom. 
Theorem 1.1. For sufficiently small $\varepsilon>0$ and $\gamma>0$ the following holds: Assume

$$
\|z(0)\| \leq \gamma \varepsilon^{n},
$$

Then, there is a constant (depending only on $n$ ) such that at time $T=\operatorname{const} \varepsilon^{-1}$.

$$
\|z(T)\| \leq \gamma \varepsilon^{n}
$$

while both $\delta(T)$ and $s(T)$ have modulus less than 1 . For all intermediate $t$, one has $\|z(t)\| \leq 2 \gamma \varepsilon^{n}$, so the trajectory never moves too far from the cylinder of breathers. Furthermore, one can find $\varphi_{1}, \vartheta_{1}, z_{1}$, with $z_{1}$ in the subspace perpendicular to the tangent space to the cylinder at $\varphi_{1}, \vartheta_{1}$, with

$$
e^{\mathbf{i}\left(T \varphi_{1}+\vartheta_{1}\right)} p\left(\varphi_{1}\right)+z_{1}=e^{\mathbf{i}(T \varphi(T)+\vartheta(T))} p(\varphi(T))+z(T),
$$

and

$$
\left\|z_{1}\right\| \leq \gamma \varepsilon^{n}
$$

Finally,

$$
\varphi_{1}-\varphi_{0}=-2 \gamma \varepsilon^{2 n-1} T+\text { h.o.t. . }
$$

Remark 1.2. The important consequence of Theorem 1.1 is the observation that the bounds propagate, so we can restart the evolution, using initial conditions $\left(\varphi_{1}, \vartheta_{1}, z_{1}\right)$ instead of $\left(\varphi_{0}, \vartheta_{0}, z_{0}\right)$, and therefore one can move to $\varphi_{2}, \vartheta_{2}, z_{2}$, and so on, with controlled bounds on $\varphi_{k}$, which apply at least as long as $\varphi_{0}-\varphi_{k} \leq \gamma \varepsilon^{n}$. Also note that the deviation from the cylinder is as shown in Fig. 1, namely, the orbit can get away from $\left\|z_{k}\right\| \leq \gamma \varepsilon^{n}$, during the times between the stopping times $k T, k=1,2, \ldots$

The remainder of the paper is devoted to the proof of Theorem 1.1. After some introductory results, the first important bound is on the linear semigroup with the very weak dissipation in Sect. 6. The generator is called $\mathcal{L}_{\varphi \gamma}$, see Eq. (6.1) and its associated bound (in Corollary 6.2). In Sect. 7, we study in detail the projection onto the complement of the tangent space to the cylinder at $(\varphi, \vartheta)$. This allows, in Sect. 8, to estimate the contraction (after time $T$ ) of the $z$-component, orthogonal to the tangent space. We do this in two steps, first we evolve $z$ while staying in the basis defined at $\varphi_{0}, \vartheta_{0}$. Then, in Sect. 9, we re-orthogonalize so that we obtain Eq. (1.5). Finally, Sect. 10 gives some more details about restarting the iterations from $\varphi_{1}, \vartheta_{1}, z_{1}$ to $\varphi_{2}, \ldots$

The precise statement will be formulated and proved as Theorem 8.2.

Remark 1.3. From the results of [1] and Eq. (1.7) one can also conclude more details about the windings of Fig. 1 . The $m^{\text {th }}$ turn finishes after a time $t_{m} \approx \sqrt{\frac{2 \pi m}{\gamma \varepsilon^{2 n-1}}}$, and the "horizontal" spacing (in $\varepsilon$ ) between the windings is $2 \sqrt{2 \pi \gamma \varepsilon^{2 n-1}}(\sqrt{m+1}-\sqrt{m})$, up to terms of higher order.

We will study Eq. (1.3) for the remainder of this paper. We will also sometimes rewrite this equation in the equivalent real form by defining $w_{j}=p_{j}+\mathbf{i} q_{j}$, which yields the system of equations, for $j=1, \ldots, n$ :

$$
\begin{aligned}
& \dot{q}_{j}=-\varepsilon(\Delta p)_{j}-p_{j}+\left(q_{j}^{2}+p_{j}^{2}\right) p_{j}-\delta_{j, n} \gamma \varepsilon q_{n}, \\
& \dot{p}_{j}=\varepsilon(\Delta q)_{j}+q_{j}-\left(q_{j}^{2}+p_{j}^{2}\right) q_{j}-\delta_{j, n} \gamma \varepsilon p_{n} .
\end{aligned}
$$




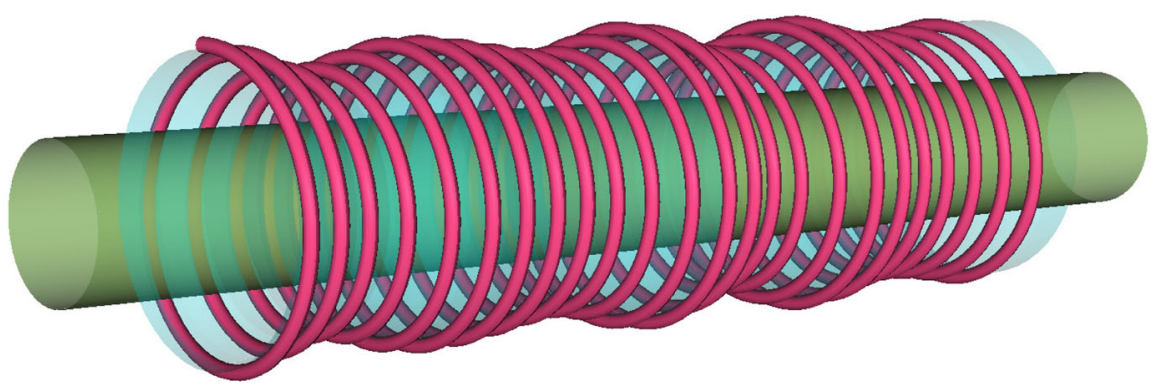

Fig. 1. Illustration of the results: Since phase space is high-dimensional, we draw the red curve in the same coordinate system as the cylinder, but it really stays in a subspace of $\mathbb{C}^{n}$ which is orthogonal to the 2-dimensional space of the cylinder. When there is no dissipation $(\gamma=0)$, then the system has a cylinder of fixed points in rotating frames (shown in green). This cylinder is parameterized by the values of $\varepsilon$, i.e., the energy of the fast coordinate $u_{1}$. When $\gamma>0$, the fixed points disappear, and instead the system hovers near the cylinder, and spiraling around it, with a phase speed of $2 \gamma \varepsilon^{2 n-1}$. We show that the orbit of all such solutions stays within a distance $\mathcal{O}\left(\varepsilon^{n}\right)$, as long as $\varepsilon$ remains small (it actually increases with time)

Note that if $\gamma=0$, this is a Hamiltonian system with:

$$
\begin{aligned}
H= & \frac{\varepsilon}{2} \sum_{j<n}\left(\left(p_{j}-p_{j+1}\right)^{2}+\left(q_{j}-q_{j+1}\right)^{2}\right) \\
& -\sum_{j=1}^{n}\left(\frac{1}{2}\left(p_{j}^{2}+q_{j}^{2}\right)-\frac{1}{4}\left(p_{j}^{2}+q_{j}^{2}\right)^{2}\right) .
\end{aligned}
$$

Finding a periodic solution of the form Eq. (1.2) (i.e., a fixed point in the rotating coordinate system) reduces to finding roots of the system of equations

$$
\begin{aligned}
& 0=-\varepsilon(\Delta p)_{j}-p_{j}+\left(q_{j}^{2}+p_{j}^{2}\right) p_{j}, \\
& 0=\varepsilon(\Delta q)_{j}+q_{j}-\left(q_{j}^{2}+p_{j}^{2}\right) q_{j} .
\end{aligned}
$$

Since we are also interested in solutions which rotate (slowly), replacing $w$ by $e^{\mathbf{i} \varphi_{0} t} w$, we study instead of Eq. (1.8) (resp. Eq. (1.10)) the related equation

$$
\begin{aligned}
& \dot{q}_{j}=-\varepsilon(\Delta p)_{j}-\left(1+\varphi_{0}\right) p_{j}+\left(q_{j}^{2}+p_{j}^{2}\right) p_{j}-\delta_{j, n} \gamma \varepsilon q_{n}, \\
& \dot{p}_{j}=\varepsilon(\Delta q)_{j}+\left(1+\varphi_{0}\right) q_{j}-\left(q_{j}^{2}+p_{j}^{2}\right) q_{j}-\delta_{j, n} \gamma \varepsilon p_{n},
\end{aligned}
$$

where the $\varphi_{0}$ dependence comes from differentiating the exponential factor $e^{\mathbf{i} \varphi_{0} t}$.

Remark 1.4. We use $\varphi_{0}$ to designate a constant rotation speed, while later, $\varphi$ will stand for a time-dependent rotation speed.

Remark 1.5. The reader who is familiar with the paper [1] can jump to Sect. 3 , since much of the material in this section and the next is basically repeated from that reference.

Theorem 1.6. Suppose that the damping coefficient $\gamma$ equals 0 in Eq. (1.11). There exist constants $\varepsilon_{*}>0, \varphi_{*}>0$, such that for $|\varepsilon|<\varepsilon_{*}$ and $\left|\varphi_{0}\right|<\varphi_{*}$, Eq. (1.11) has a periodic solution of the form $w_{j}\left(t ; \varphi_{0}\right)=e^{\mathbf{i} t \varphi_{0}} p_{j}\left(\varphi_{0}\right)$, with $p_{1}\left(\varphi_{0}\right)=1+\mathcal{O}\left(\varepsilon, \varphi_{0}\right)$, and $p_{j}\left(\varphi_{0}\right)=\mathcal{O}\left(\varepsilon^{j-1}\right)$ for $j=2, \ldots, n$. 
Proof. If we insert $w_{j}\left(t ; \varphi_{0}\right)=e^{\mathbf{i} t \varphi_{0}} p_{j}\left(\varphi_{0}\right)$ into Eq. (1.3), and take real and imaginary parts, we find that the amplitudes $p \in \mathbb{R}^{n}$ of these periodic orbits are (for $\gamma=0$ ) solutions of

$$
F_{j}\left(p ; \varphi_{0}, \varepsilon\right)=-\varepsilon(\Delta p)_{j}-\left(1+\varphi_{0}\right) p_{j}+p_{j}^{3}=0, \quad j=1, \ldots, n .
$$

Setting $p_{j}^{0}=\delta_{j, 1}$, we have

$$
F_{j}\left(p^{0} ; 0,0\right)=0,
$$

for all $j$. Furthermore, the Jacobian matrix at this point is the diagonal matrix

$$
\left(D_{p} F\left(p^{0} ; 0,0\right)\right)_{i, j}=\left(3 \delta_{i, 1}-1\right) \delta_{i, j}
$$

which is obviously invertible.

Thus, by the implicit function theorem, for $\left(\varphi_{0}, \varepsilon\right)$ in some neighborhood of the origin, Eq. (1.12) has a unique fixed point $p=p\left(\varphi_{0}, \varepsilon\right)$, and since $F$ depends analytically on $\left(\varphi_{0}, \varepsilon\right)$, so does $p\left(\varphi_{0}, \varepsilon\right)$.

It is easy to compute the first few terms of this fixed point:

$$
\begin{aligned}
p_{1} & =1+\frac{1}{2}\left(\varphi_{0}-\varepsilon\right)+\mathcal{O}_{2}, \\
p_{2} & =-\varepsilon+\mathcal{O}_{2}, \\
p_{3} & =\varepsilon^{2}+\mathcal{O}_{3}, \\
& \cdots \\
p_{j} & =(-1)^{j-1} \varepsilon^{j}+\mathcal{O}_{j+1},
\end{aligned}
$$

where $\mathcal{O}_{k}$ denotes terms of order $k$ in $\varphi_{0}, \varepsilon$ together.

Remark 1.7. Since Eq. (1.3) is invariant under complex rotations $w_{j} \rightarrow e^{\mathbf{i} \vartheta_{0}} w_{j}$, we actually have a circle of fixed points (when $\gamma=0$ ). However, these are the only fixed points with $\left|w_{1}\right| \approx 1$. We will continue with $\vartheta_{0}=0$, and reintroduce $\vartheta_{0} \neq 0$ only in Sect. 3.

\section{The Eigenspace of the Eigenvalue 0}

Consider the linearization of the system Eq. (1.11) around the periodic orbit (fixed point) we found in Theorem 1.6. Denote by $Z_{*}$ this solution,

$$
Z_{*}=\left(p_{1}, p_{2}, \ldots, p_{n}, q_{1}, q_{2}, \ldots, q_{n}\right)^{\top},
$$

where $q_{j}=0$ and $p_{j}=p_{j}\left(\varphi_{0}, \varepsilon\right)$ as found in Theorem 1.6. In order to avoid overburdening the notation, we will write out the formulas which follow for the case $n=3$ - the expressions for general (finite) values of $n$ are very similar. We also omit the $\varepsilon$ dependence from $p\left(\varphi_{0}, \varepsilon\right)$. The linearization of the evolution Eq. (1.11) at $Z_{*}$ leads (for $\gamma=0$ ) to an equation of the form

$$
\frac{\mathrm{d} x}{\mathrm{~d} t}=M_{\varphi_{0}, \varepsilon} x=\left(\begin{array}{cc}
0 & A_{\varphi_{0}, \varepsilon} \\
B_{\varphi_{0}, \varepsilon} & 0
\end{array}\right) x,
$$


and with $1_{\varphi_{0}} \equiv\left(1+\varphi_{0}\right)$ :

$$
A_{\varphi_{0}, \varepsilon}=\left(\begin{array}{ccc}
1_{\varphi_{0}}-\varepsilon-\left(p_{1}\right)^{2} & \varepsilon & 0 \\
\varepsilon & 1_{\varphi_{0}}-2 \varepsilon-\left(p_{2}\right)^{2} & \varepsilon \\
0 & \varepsilon & 1_{\varphi_{0}}-\varepsilon-\left(p_{3}\right)^{2}
\end{array}\right),
$$

where the $p_{j}=p\left(\varphi_{0}\right)_{j}$ are the stationary solutions of Eq. (1.11). Similarly,

$$
B_{\varphi_{0}, \varepsilon}=\left(\begin{array}{ccc}
-1_{\varphi_{0}}+\varepsilon+3\left(p_{1}\right)^{2} & -\varepsilon & 0 \\
-\varepsilon & -1_{\varphi_{0}}+2 \varepsilon+3\left(p_{2}\right)^{2} & -\varepsilon \\
0 & -\varepsilon & -1_{\varphi_{0}}+\varepsilon+3\left(p_{3}\right)^{2}
\end{array}\right)
$$

Similar expressions hold for other values of $n$.

Among the key facts that we will establish below is that $M_{\varphi_{0}, \varepsilon}$ has a two-dimensional zero eigenspace, with an explicitly computable basis, for all values of $\varepsilon$. Then, in subsequent sections we will show that the remainder of the spectrum lies on the imaginary axis and that all non-zero eigenvalues are simple and separated from the remainder of the spectrum of $M_{\varphi_{0}, \varepsilon}$ by a distance at least $C \varepsilon$. All of these facts turn out to be essential for our subsequent calculations and establishing them is complicated by the extreme degeneracy of the eigenvalues of $M_{\varphi_{0}, 0}$ about which we wish to perturb.

The following lemma will allow to simplify notation:

Lemma 2.1. One has the identity

$$
\partial_{\varphi} p\left(\varphi_{0}\right)=B_{\varphi_{0}, \varepsilon}^{-1} p\left(\varphi_{0}\right)
$$

Proof. This follows by differentiating Eq. (1.12) and comparing to the definition of $B_{\varphi_{0}, \varepsilon}$ in Eq. (2.2).

Lemma 2.2. Define $B_{\varphi_{0}, \varepsilon}=L-\varphi_{0} \mathbb{1}+3\left(p(\varphi)_{0}\right)^{2}$, with $L=\varepsilon \Delta-\mathbb{1}$. That is, we view $B_{\varphi_{0}, \varepsilon}$ as a real $n \times n$ matrix and $\left(p\left(\varphi_{0}\right)\right)^{2}$ as the diagonal matrix with components $\left(\left(p\left(\varphi_{0}\right)_{1}\right)^{2}, \ldots,\left(p\left(\varphi_{0}\right)_{n}\right)^{2}\right)$. Then the zero eigenspace of the matrix $M_{\varphi_{0}, \varepsilon}$ is spanned by the 2 -component vectors

$$
\begin{aligned}
& v_{\varphi_{0}}^{(1)}=\left(\begin{array}{c}
0 \\
p\left(\varphi_{0}\right)
\end{array}\right), \\
& v_{\varphi_{0}}^{(2)}=\left(\begin{array}{c}
B_{\varphi_{0}, \varepsilon}^{-1} p\left(\varphi_{0}\right) \\
0
\end{array}\right),
\end{aligned}
$$

Proof. To see that $M_{\varphi_{0}, \varepsilon} v_{\varphi_{0}}^{(1)}=0$, note that Eq. (1.1) is invariant under $u \rightarrow e^{\mathbf{i} \vartheta} u$. Thus, viewed in $\mathbb{C}^{n}$, the quantity $e^{\mathbf{i} \vartheta}\left(p\left(\varphi_{0}, \varepsilon\right)+\mathbf{i} 0\right)$ is a solution for all $\vartheta$. Taking the derivative w.r.t. $\vartheta$, at $\vartheta=0$ and considering the real and imaginary parts of the resulting equation shows that $v_{\varphi_{0}}^{(1)}$ is a solution of $M_{\varphi_{0}, \varepsilon} v_{\varphi_{0}}^{(1)}=0$. From the form of $M_{\varphi_{0}, \varepsilon}$ and the invertibility of $B_{\varphi_{0}, \varepsilon}$ we see immediately that $v_{\varphi_{0}}^{(2)}$ is mapped onto the direction of $v_{\varphi_{0}}^{(1)}$.

We will also need the adjoint eigenvectors of $M$ : 
Lemma 2.3. The adjoint eigenvectors are given by

$$
\begin{aligned}
& n_{\varphi_{0}}^{(1)}=\left(2+\mathcal{O}\left(\varphi_{0}, \varepsilon\right)\right) \cdot\left(0, B_{\varphi_{0}}^{-1} p\left(\varphi_{0}\right)\right)^{\top}, \\
& n_{\varphi_{0}}^{(2)}=\left(2+\mathcal{O}\left(\varphi_{0}, \varepsilon\right)\right) \cdot\left(p\left(\varphi_{0}\right), 0\right)^{\top} .
\end{aligned}
$$

They are normalized to satisfy

$$
\begin{gathered}
\left\langle n_{\varphi_{0}}^{(1)} \mid v_{\varphi_{0}}^{(1)}\right\rangle=\left\langle n_{\varphi_{0}}^{(2)} \mid v_{\varphi_{0}}^{(2)}\right\rangle=1, \\
\left\langle n_{\varphi_{0}}^{(2)} \mid v_{\varphi_{0}}^{(1)}\right\rangle=\left\langle n_{\varphi_{0}}^{(1)} \mid v_{\varphi_{0}}^{(2)}\right\rangle=0 .
\end{gathered}
$$

Remark 2.4. The approximate versions are

$$
\begin{aligned}
& n_{\varphi_{0}}^{(1)} \sim(0, \ldots, 0,1,0, \ldots, 0)^{\top}, \\
& n_{\varphi_{0}}^{(2)} \sim(2,0, \ldots, 0)^{\top} .
\end{aligned}
$$

Proof. Because of the block form of $M$ and the fact that $A$ and $B$ are symmetric, we have

$$
M_{\varphi_{0}, \varepsilon}^{*}=\left(\begin{array}{cc}
0 & B_{\varphi_{0}, \varepsilon} \\
A_{\varphi_{0}, \varepsilon} & 0
\end{array}\right) .
$$

But then, since we know from the computation of the eigenvectors of $M$ that $B_{\varphi_{0}, \varepsilon} p\left(\varphi_{0}\right)=$ 0 , we can check immediately that

$$
\tilde{n}_{\varphi_{0}}^{(2)}=\left(p\left(\varphi_{0}\right), 0\right)^{\top}
$$

satisfies $M^{*} \tilde{n}_{\varphi_{0}}^{(2)}=0$. Likewise,

$$
\tilde{n}_{\varphi_{0}}^{(1)}=\left(0, B_{\varphi_{0}, \varepsilon}^{-1} p\right)^{\top}
$$

satisfies

$$
M_{\varphi_{0}, \varepsilon}^{*} \tilde{n}_{\varphi_{0}}^{(1)}=\left(p\left(\varphi_{0}\right), 0\right)^{\top}=\tilde{n}_{\varphi_{0}}^{(2)} .
$$

Thus, $\tilde{n}_{\varphi_{0}}^{(1)}$ and $\tilde{n}_{\varphi_{0}}^{(2)}$ span the zero eigenspace of the adjoint matrix. The normalization is checked from the definitions.

\section{Evolution Equations for $\boldsymbol{\gamma}>0$}

Consider Eq. (1.3), with dissipation: Here, $C_{\Gamma}$ is not a scalar, but a diagonal matrix, whose diagonal will be taken as $(0,0, \ldots, \gamma \varepsilon) \in \mathbb{C}^{n}$. Thus, our evolution equation is

$$
-\mathbf{i} \dot{W}=L W+|W|^{2} W+\mathbf{i} C_{\Gamma} W
$$

with $L=\varepsilon \Delta-\mathbb{1}$, as before. We are interested in the time dependence of two real "slow" variables which we call $\varphi(t)$ and $\vartheta(t)$, and so we set

$$
W(t)=e^{\mathbf{i}(t \varphi(t)+\vartheta(t))}(p(\varphi(t))+z(t)), \quad W, z \in \mathbb{C}^{n} .
$$

Remark 3.1. Recall that the notation $\varphi_{0}$ stands for a constant phase speed, while $\varphi=\varphi(t)$ will always mean a time-dependent quantity. 
Our decomposition is inspired by modulation theory approaches to study the stability of solitary waves and patterns with respect to perturbations [7-10]. In particular, we will choose the initial decomposition of the solution so that the initial value of $z(0)$ lies in the subspace conjugate to the zero-eigenspace of the linearization. We then prove (somewhat surprisingly) that all modes orthogonal to the zero subspace are uniformly damped which allows us to show that the values of $z(t)$ remain bounded for very long times. Omitting the arguments $(t)$, we find

$$
\begin{aligned}
\dot{W}= & \mathbf{i}(\varphi+t \dot{\varphi}+\dot{\vartheta}) e^{\mathbf{i}(t \varphi+\vartheta)}(p(\varphi)+z) \\
& +e^{\mathbf{i}(t \varphi+\vartheta)}\left(\partial_{\varphi} p(\varphi) \dot{\varphi}+\dot{z}\right) .
\end{aligned}
$$

Then Eq. (3.1) leads to (using again that powers and products are taken componentwise),

$$
\begin{aligned}
(\varphi+ & t \dot{\varphi}+\dot{\vartheta}) e^{\mathbf{i}(t \varphi+\vartheta)}(p(\varphi)+z)-\mathbf{i} e^{\mathbf{i}(t \varphi+\vartheta)}\left(\partial_{\varphi} p(\varphi) \dot{\varphi}+\dot{z}\right) \\
= & e^{\mathbf{i}(t \varphi+\vartheta)} L(p(\varphi))+e^{\mathbf{i}(t \varphi+\vartheta)} L z \\
& +\left(e^{\mathbf{i}(t \varphi+\vartheta)}(p(\varphi)+z)\right)^{2}\left(e^{-\mathbf{i}(t \varphi+\vartheta)}(p(\varphi)+\bar{z})\right)+\mathbf{i} C_{\Gamma} e^{\mathbf{i}(t \varphi+\vartheta)}(p(\varphi)+z) .
\end{aligned}
$$

The factors of $e^{\mathbf{i}(t \varphi+\vartheta)}$ cancel and we get

$$
\begin{aligned}
(\varphi & +t \dot{\varphi}+\dot{\vartheta})(p(\varphi)+z)-\mathbf{i}\left(\partial_{\varphi} p(\varphi) \dot{\varphi}+\dot{z}\right) \\
& =L(p(\varphi))+L z+(p(\varphi)+z)^{2}(p(\varphi)+\bar{z})+\mathbf{i} C_{\Gamma}(p(\varphi)+z) .
\end{aligned}
$$

We now expand this equation to first order in $z$ and this leads to

$$
\begin{aligned}
(\varphi+ & t \dot{\varphi}+\dot{\vartheta})(p(\varphi)+z)-\mathbf{i}\left(\partial_{\varphi} p(\varphi) \dot{\varphi}+\dot{z}\right) \\
= & L(p(\varphi))+L z \\
& +(p(\varphi))^{3}+2(p(\varphi))^{2} z+p(\varphi) \bar{z} \\
& +\mathbf{i} C_{\Gamma}(p(\varphi)+z)+\mathcal{O}\left(|z|^{2}\right) .
\end{aligned}
$$

Set now $z=\xi+\mathbf{i} \eta$.

In what follows, we will switch back and forth between the real and complex representations of the solutions and will refer to $z=\xi+\mathbf{i} \eta \in \mathbb{C}^{n}$ and $\zeta=(\xi, \eta) \in \mathbb{R}^{2 n}$ interchangeably, allowing the context to distinguish between the two ways of writing the solution. When we consider $\xi$ and $\eta$, which are $n$ dimensional vectors, one should note that $\xi=\left(\xi_{1}, \ldots, \xi_{n}\right)^{\top}$ while $\eta=\left(\eta_{1}, \ldots, \eta_{n}\right)^{\top}$. At various points in the argument, will use restrictions of our equations to these two spaces which we call $\mathbb{P}_{\varphi_{0}}^{\xi}$ and $\mathbb{P}_{\varphi_{0}}^{\eta}$.

Taking the real and imaginary components of Eq. (3.3), we obtain the following equations in $\mathbb{R}^{n}$ :

$$
\begin{aligned}
& (t \dot{\varphi}+\dot{\vartheta})(p(\varphi)+\xi)+\dot{\eta}=(L-\varphi) \xi+3(p(\varphi))^{2} \xi-C_{\Gamma} \eta+\mathcal{O}_{2}, \\
& (t \dot{\varphi}+\dot{\vartheta}) \eta-\left(\partial_{\varphi} p(\varphi) \dot{\varphi}-\dot{\xi}=(L-\varphi) \eta+(p(\varphi))^{2} \eta+C_{\Gamma}(p(\varphi)+\xi)+\mathcal{O}_{2},\right.
\end{aligned}
$$

where $\mathcal{O}_{2}$ refers to terms that are at least quadratic in $(\xi, \eta)$.

We next study what happens in the complement of the two-dimensional zero eigenspace identified at the end of the previous section, when one adds dissipation on the coordinate $n$. In the standard basis, when $n=3$, the dissipation is given, as before, by

$$
C_{\Gamma}=\left(\begin{array}{ccc}
0 & 0 & 0 \\
0 & 0 & 0 \\
0 & 0 & \gamma \varepsilon
\end{array}\right) .
$$


In the full space, we have the $2 n \times 2 n$ matrix

$$
\Gamma=\left(\begin{array}{cc}
C_{\Gamma} & 0 \\
0 & C_{\Gamma}
\end{array}\right) .
$$

We fix a $\varphi(0)=\varphi_{0}$ and we consider the projection

$$
\mathbb{P}=\mathbb{P}_{\varphi_{0}}=\mathbb{1}-\left|v_{\varphi_{0}}^{(1)}\right\rangle\left\langle n_{\varphi_{0}}^{(1)}|-| v_{\varphi_{0}}^{(2)}\right\rangle\left\langle n_{\varphi_{0}}^{(2)}\right| .
$$

This is the projection onto the complement of the space spanned by the 0 eigenvalue.

We will require that $\zeta=(\xi, \eta)$ remains in the range of $\mathbb{P}_{\varphi_{0}}$. As time passes, the base point $(\varphi, \vartheta)$ will change, and this will lead to secular growth in $\zeta$, an issue which we discuss in detail below.

We rearrange Eq. (3.4) as

$$
\begin{aligned}
\dot{\xi}= & \mathbb{P}_{\varphi_{0}}^{\xi}\left(\left(-(L-\varphi)-(p(\varphi))^{2}\right) \eta\right. \\
& \left.-C_{\Gamma} \xi+(t \dot{\varphi}+\dot{\vartheta}) \eta-\partial_{\varphi}(p(\varphi)) \dot{\varphi}-C_{\Gamma} p(\varphi)+\mathcal{O}_{2}\right), \\
\dot{\eta}= & \mathbb{P}_{\varphi_{0}}^{\eta}\left(\left((L-\varphi)+3(p(\varphi))^{2}\right) \xi-C_{\Gamma} \eta-(t \dot{\varphi}+\dot{\vartheta})(p(\varphi)+\xi)+\mathcal{O}_{2}\right) .
\end{aligned}
$$

We will compute these projections in detail in Sect. 7.

\section{Spectral Properties of the Linearization at $\gamma=0$}

In this section, we consider the action of the matrix $M_{\varphi_{0}, \varepsilon}$, when projected (with $\mathbb{P}_{\varphi_{0}}$ ) onto the complement of the subspace associated with the 0 eigenspace. Recall from above that:

$$
\mathbb{P}_{\varphi_{0}}=\mathbb{1}-\left|v_{\varphi_{0}}^{(1)}\right\rangle\left\langle n_{\varphi_{0}}^{(1)}|-| v_{\varphi_{0}}^{(2)}\right\rangle\left\langle n_{\varphi_{0}}^{(2)}\right| .
$$

We will see that this projection is very close to the projection onto the complement of the 1 st and $(n+1)$ st component of the vectors in $\mathbb{R}^{2 n}$.

We use perturbation theory, starting from the matrix $M_{\varphi_{0}=0, \varepsilon=0}$. We write the formulas for $n=4$. The discussion starts with $\varepsilon=\varphi_{0}=0$. Then, we have the quantities

$$
A_{0,0}=A_{\varphi_{0}=0, \varepsilon=0}\left(\begin{array}{l|l|ll}
0 & 0 & 0 & 0 \\
\hline 0 & 1 & 0 & 0 \\
0 & 0 & 1 & 0 \\
0 & 0 & 0 & 1
\end{array}\right)=\left(\begin{array}{l|l}
0 & \\
\hline & \mathbb{1}
\end{array}\right),
$$

and

$$
B_{0,0}=B_{\varphi=0, \varepsilon=0}=\left(\begin{array}{r|rrr}
2 & 0 & 0 & 0 \\
\hline 0 & -1 & 0 & 0 \\
0 & 0 & -1 & 0 \\
0 & 0 & 0 & -1
\end{array}\right)=\left(\begin{array}{l|l}
2 & \\
\hline & -\mathbb{1}
\end{array}\right),
$$

which follow by substitution. We set

$$
M_{0,0} \equiv\left(\begin{array}{cc}
0 & A_{0,0} \\
B_{0,0} & 0
\end{array}\right),
$$


and study first the spectrum of $M_{0,0}$. The spectrum (and the eigenspaces) of $M_{\varphi, \varepsilon}$ will then be shown to be close to that of $M_{0,0}$.

The eigenvalues of $M_{0,0}$ are: A double 0 , and $n-1$ pairs of eigenvalues $\pm \mathbf{i}$. When $n=4$, the corresponding eigenvectors are:

$$
\begin{aligned}
e^{(1)} & =(0,0,0,0,1,0,0,0)^{\top}, \\
e^{(3),(4)} & =(0, \pm \mathbf{i}, 0,0,0,1,0,0)^{\top}, \\
e^{(5),(6)} & =(0,0, \pm \mathbf{i}, 0,0,0,1,0)^{\top}, \\
e^{(7),(8)} & =(0,0,0, \pm \mathbf{i}, 0,0,0,1)^{\top} .
\end{aligned}
$$

Note that $e^{(2)}$ is missing, but the vector $e^{(2)}=(1,0,0,0,0,0,0,0)^{\top}$ is mapped onto $2 e^{(1)}$ and so $e^{(1)}$ and $e^{(2)}$ span the 0 eigenspace.

Since we have a symplectic problem (when $\gamma=0$ ), we need to do the calculations in an appropriate basis. This is inspired by the paper [11]. The coordinate transformation is defined by the following matrix: Let $s=1 / \sqrt{2}$, and define (for the case $n=4$ ),

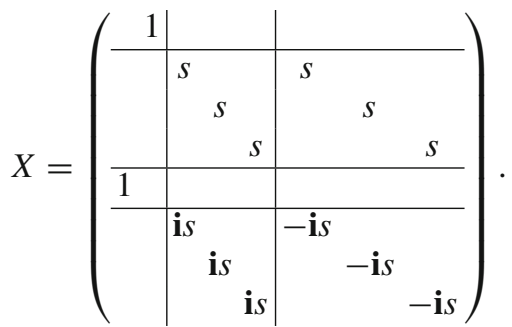

The columns are the normalized eigenvectors of our problem, for $\varepsilon=\varphi=0$. Empty positions are " 0 "s and the second vector is mapped on the first (up to a factor of 2). With our choice of $s$ we have $X^{*} X=1$, where $X^{*}$ is the Hermitian conjugate of $X$.

Definition 4.1. If $Y$ is a matrix, we write its transform as $\mathcal{X}(Y)=X^{*} Y X$.

In the new basis, we get:

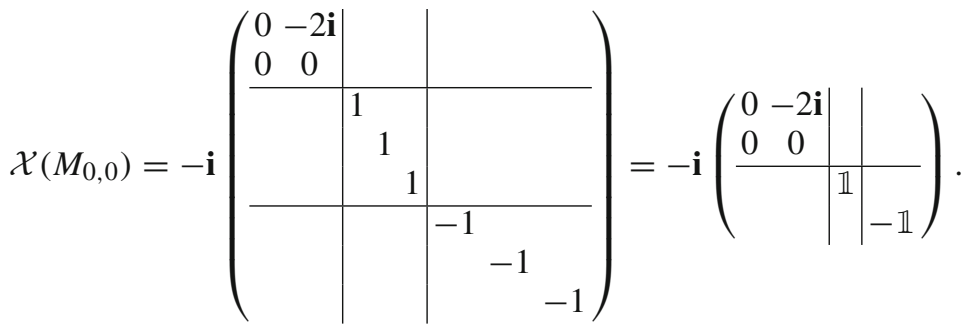

Therefore, we have diagonalized $M_{0,0}$ up to its nilpotent block, and we also see that the other parts of $\mathcal{X}\left(M_{0,0}\right)$ are imaginary, which reflects the symplectic nature of the model.

We now turn to the case of $M_{\varphi_{0}, \varepsilon}$ which we view as a perturbation of $M_{0,0}$ in the following way:

$$
M_{\varphi_{0}, \varepsilon}=M_{\varphi_{0}, 0}+E_{1}+E_{2} \text {, }
$$

where

$$
M_{\varphi_{0}, 0}=\left(\begin{array}{cc}
0 & A_{\varphi_{0}, 0} \\
B_{\varphi_{0}, 0} & 0
\end{array}\right),
$$


with

$$
\begin{aligned}
A_{\varphi_{0}, 0} & =\operatorname{diag}\left(0,1_{\varphi_{0}}, \ldots, 1_{\varphi_{0}}\right), \\
B_{\varphi_{0} .0} & =\operatorname{diag}\left(-2 \cdot 1_{\varphi_{0}},-1_{\varphi_{0}}, \ldots,-1_{\varphi_{0}}\right) .
\end{aligned}
$$

The matrix $E_{1}$ collects the terms in $M_{\varphi_{0}, \varepsilon}$ which are linear in $\varepsilon$, while $E_{2}$ collects all higher order terms. The matrix $E_{1}$ is easily derived from Eqs. (1.13) and (4.1)-(4.2) ${ }^{2}$ :

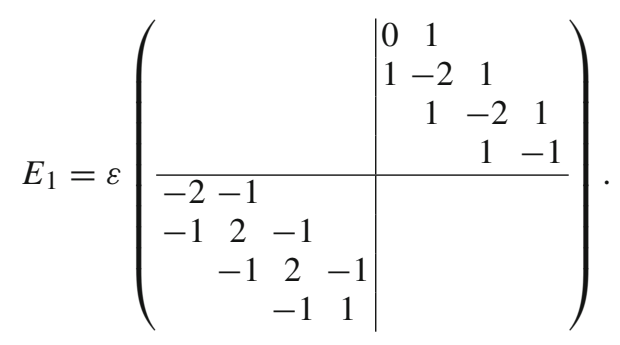

We now apply the coordinate transformation to $M_{\varphi_{0}, 0}$ and $E_{1}$. We first observe that

$$
\mathcal{X}\left(M_{\varphi_{0}, 0}\right)=\left(1+\varphi_{0}\right) \mathcal{X}\left(M_{0,0}\right) \text {. }
$$

Applying the transformation to $E_{1}$, one gets (using again $s=1 / \sqrt{2}$ ):

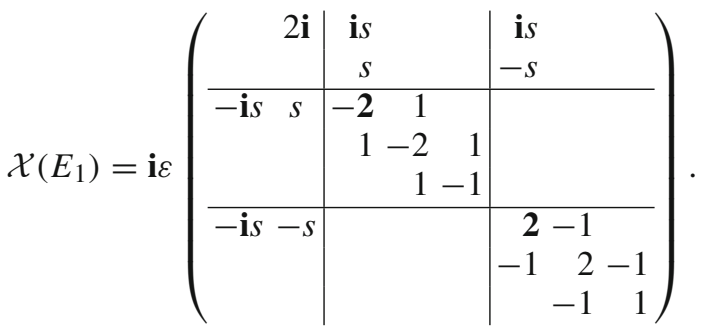

The reader should be aware that the seeming irregularities of the matrix $\mathcal{X}\left(E_{1}\right)$ are due to the differences between the expansions of $p_{1}$ and the other $p_{j}$ in powers of $\varepsilon$. We next split $\mathcal{X}\left(E_{1}\right)=\mathcal{X}\left(E_{11}\right)+\mathcal{X}\left(E_{12}\right)$, where $^{3}$

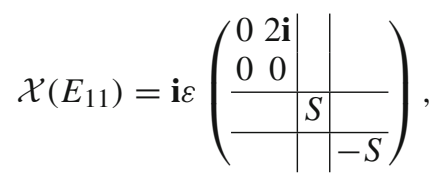

and the $\pm S$ are the tri-diagonal parts of Eq. (4.6). It is important to observe that the $S$ is tri-diagonal, symmetric, with non-zero off-diagonal elements. Clearly, $\mathcal{X}\left(E_{12}\right)$ contains

\footnotetext{
2 The generalization to arbitrary $n$ is obtained by "filling in" more rows of the form $1,-2,1$ resp. $-1,2,-1$, while retaining the first and last rows.

${ }^{3}$ We maintain the somewhat redundant notation $\mathcal{X}(\cdot)$ so that the reader immediately sees on which space the object in question acts.
} 
only the two top rows and the first two columns of $\mathcal{X}\left(E_{1}\right)$. It is thus of the form

$$
\mathcal{X}\left(E_{12}\right)=\mathbf{i} \varepsilon\left(\begin{array}{cc|c|c}
0 & 0 & * * * & * * * \\
0 & 0 & * * * & * * * \\
* & * & & \\
* & * & & \\
* & * & & \\
\hline * & * & & \\
* & * & & \\
* & * & &
\end{array}\right),
$$

where the "*" denote elements of at most $\mathcal{O}_{1}$, cf. Eq. (4.6).

All in all, we have decomposed

$$
\mathcal{X}\left(M_{\varphi_{0}, \varepsilon}\right)=\left(1+\varphi_{0}\right) \mathcal{X}\left(M_{0,0}\right)+\mathcal{X}\left(E_{11}\right)+\mathcal{X}\left(E_{12}\right)+\mathcal{X}\left(E_{2}\right)
$$

The term $\mathcal{X}\left(E_{2}\right)$ contributes to second order, and it only remains to understand the role of $\mathcal{X}\left(E_{12}\right)$. Note now that $\mathcal{X}\left(E_{12}\right)$, which is of the form of Eq. (4.8), couples the 0 block to $S$ and $-S$, but only to the first component of these matrices. ${ }^{4}$ The following argument from classical perturbation theory shows that this can only contribute to second order in $\varepsilon$ to the spectrum.

The first order shift of an eigenvalue close to $\mathbf{i}$ with eigenvector $v$ is simply $\left\langle v \mid \mathcal{X}\left(E_{12}\right) v\right\rangle$. But, $v$ is of the form

$$
v=\left(0,0, v_{1}, v_{2}, v_{3}, 0,0,0\right)^{\top},
$$

due to the form $\mathcal{X}\left(M_{\varphi_{0}, 0}+E_{11}\right)$. Thus, $\mathcal{X}\left(E_{12}\right) v$ is of the form

$$
\mathcal{X}\left(E_{12}\right) v=(*, *, 0,0,0,0,0,0)^{\top},
$$

where "*" denotes possibly non-zero elements. From the form of $v$, this implies that

$$
\left\langle v \mid \mathcal{X}\left(E_{12}\right) v\right\rangle=0 \text {. }
$$

This means that $\mathcal{X}\left(E_{12}\right)$ contributes only in second order in $\varepsilon$ to the eigenvalues.

\section{Complement of the Zero Eigenspace}

Recall that our goal is to write the solution of (1.3) as

$$
w_{j}(t)=e^{\mathbf{i}(\varphi(t) t+\vartheta(t))}\left(p_{j}(\varphi(t))+z_{j}(t)\right),
$$

and then follow the evolution of $\varphi, \vartheta$, and $z=(\xi+\mathbf{i} \eta)$. Since $\zeta(t)=(\xi(t), \eta(t))$ is constructed to lie in the subspace orthogonal to the tangent space of the cylinder of breathers at $\left(\varphi_{0}, \vartheta_{0}\right)$, we construct the projection $\mathbb{P}$ onto the tangent space at $\varphi_{0}, \vartheta_{0}$. We show that, somewhat surprisingly, with the exception of the zero eigenspace, all other eigenvalues of the linearized matrix are simple, lie on the imaginary axis, and are separated by a distance of at least $C \varepsilon$ from the remainder of the spectrum. It is convenient to work directly with the transformation $\mathcal{X}(\cdot)$ of Eq. (4.3).

\footnotetext{
4 This is a remnant of the nearest neighbor coupling of the model.
} 
Theorem 5.1. The operator $\mathcal{X}\left(\mathbb{P} M_{\varphi_{0}, \varepsilon}\right)$ has two eigenvalues which are within $\mathcal{O}(\varepsilon)$ of 0 , and $(n-1)$ purely imaginary eigenvalues close to $\mathbf{i}$, separated by $C \varepsilon$, with $C>0$. The corresponding eigenvectors (in the $\mathcal{X}(\cdot)$ basis) are orthogonal. Furthermore, these eigenvalues have non-vanishing last component (i.e., the components $2+(n-1)$ and $2+2(n-1)$ in the $\mathcal{X}(\cdot)$ representation). Analogous statements hold for the $n-1$ eigenvalues near $-\mathbf{i}$.

Proof. The remainder of this section is devoted to the proof of this theorem.

A calculation (using our formulas for $v^{(j)}, n^{(j)}$ ) shows that

$$
\mathcal{X}(\mathbb{P})=\mathcal{X}\left(\mathbb{P}_{0}\right)+\mathcal{X}\left(\mathbb{P}_{1}\right)+\mathcal{X}\left(\mathbb{P}_{2}\right)
$$

where



where the orders of the elements of $P_{j}$ are

$$
P_{j}=\left(\begin{array}{lll}
\varepsilon^{2} & \varepsilon^{3} & \varepsilon^{4} \\
\varepsilon^{3} & \varepsilon^{4} & \varepsilon^{5} \\
\varepsilon^{4} & \varepsilon^{5} & \varepsilon^{6}
\end{array}\right) .
$$

Furthermore the $P_{j}$ are symmetric. Note that $\left(P_{j}\right)_{i, k}=\mathcal{O}\left(\varepsilon^{i+k}\right)$. Finally, showing orders only,

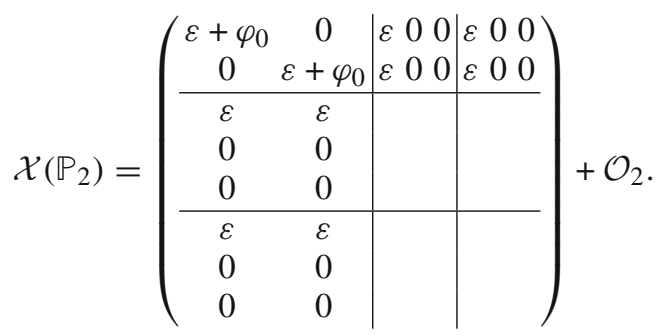

The omitted terms are similar to those in $P_{j}$ (again a symmetric matrix). Therefore, the eigenvectors are orthogonal. (This actually follows also from the Hamiltonian nature of the problem, but we need more information to control the $\gamma$-dependence.)

Remark 5.2. Clearly, $\mathcal{X}\left(\mathbb{P}_{0}\right)$ is the projection on the eigenspace spanned by $\pm \mathbf{i}$, when $\varepsilon=0$. The part $\mathcal{X}\left(\mathbb{P}_{1}\right)$ contains the couplings within the subspace of the eigenvalues $\pm \mathbf{i}$, while $\mathcal{X}\left(\mathbb{P}_{2}\right)$ describes the coupling between the zero-eigenspace of dimension 2 and its complement.

From Sect. 4 we also have the decomposition Eq. (4.9):

$$
\mathcal{X}\left(M_{\varphi_{0}, \varepsilon}\right)=\left(1+\varphi_{0}\right) \mathcal{X}\left(M_{0,0}\right)+\mathcal{X}\left(E_{11}\right)+\mathcal{X}\left(E_{12}\right)+\mathcal{X}\left(E_{2}\right)
$$


Combining with Eq. (5.1), we see that

$$
\mathcal{X}\left(\mathbb{P} M_{\varphi_{0}, \varepsilon}\right)=\mathcal{X}(\mathbb{P}) \mathcal{X}\left(M_{\varphi_{0}, \varepsilon}\right)
$$

leads to 12 terms, many of which are of second order in $\varepsilon$ and $\varphi_{0}$. We start with the dominant ones.

Since $\mathcal{X}\left(\mathbb{P}_{0}\right)$ is just the projection onto the complement of the first 2 eigendirections, we get from Eq. (4.4),

$$
\begin{aligned}
& \mathcal{X}\left(\mathbb{P}_{0} M_{\varphi_{0}, 0}\right)=\mathcal{X}\left(\mathbb{P}_{0}\right) \mathcal{X}\left(M_{\varphi_{0}, 0}\right) \\
& =\mathbf{i} 1_{\varphi}\left(\begin{array}{ll|l|l}
\hline & 0 & & \\
\hline & & \mathbb{1} & \\
\hline & & \mathbb{1}
\end{array}\right)\left(\begin{array}{cc|c|c}
0 & -2 \mathbf{i} & \mid & \\
0 & 0 & & \\
\hline & & \mathbb{1} & \\
\hline & & -\mathbb{1}
\end{array}\right)=-\mathbf{i} 1_{\varphi}\left(\begin{array}{ll|l|l}
0 & 0 & \\
\hline & 0 & & \\
\hline & \mathbb{1} & \\
\hline & & -\mathbb{1}
\end{array}\right),
\end{aligned}
$$

where $1_{\varphi} \equiv 1+\varphi$. This is clearly the leading constant term.

The next term is at the origin of the $\varepsilon$-splitting of Theorem 5.1. Using Eq. (4.7), we get

$$
\begin{aligned}
& \mathcal{X}\left(\mathbb{P}_{0} E_{11}\right)=\mathcal{X}\left(\mathbb{P}_{0}\right) \mathcal{X}\left(E_{11}\right) \\
& =\mathbf{i} \varepsilon\left(\begin{array}{ll|l|l}
0 & 0 & & \\
0 & 0 & & \\
\hline & \mathbb{1} & \\
\hline & & \mathbb{1}
\end{array}\right)\left(\begin{array}{ll|l|l}
0 & 2 \mathbf{i} & & \\
0 & 0 & & \\
\hline & S & \\
\hline & & -S
\end{array}\right)=\mathbf{i} \varepsilon\left(\begin{array}{ll|l|l}
0 & 0 & & \\
\hline 0 & 0 & & \\
\hline & S & \\
\hline & & -S
\end{array}\right) .
\end{aligned}
$$

Thus, to leading order, we find

$$
\mathcal{X}\left(\mathbb{P}_{0}\left(M_{\varphi_{0}, 0}+E_{11}\right)\right)=-\mathbf{i} 1_{\varphi}\left(\begin{array}{ll|l|l}
0 & 0 & & \\
0 & 0 & & \\
\hline & \mathbb{1} & \\
\hline & & -\mathbb{1}
\end{array}\right)+\mathbf{i} \varepsilon\left(\begin{array}{ll|l|l}
0 & 0 & \mid & \\
0 & 0 & & \\
\hline & S & \\
\hline & & -S
\end{array}\right) .
$$

We now use

Proposition 5.3. Consider a tri-diagonal matrix $U$ with $U_{i, i+1}=U_{i, i-1} \neq 0$ for all $i$ and arbitrary elements in the diagonal. Then

\section{All eigenfunctions of $U$ have their first and last components non-zero}

2. All eigenvalues of $U$ are simple.

Postponing the proof, we conclude, by applying the proposition to $S$ and $-S$ separately, that: The matrix $\mathcal{X}\left(\mathbb{P}_{0} E_{11}\right)$ has a double eigenvalue 0 , and $2(n-1)$ simple, purely imaginary, eigenvalues $\pm \lambda_{1}, \ldots, \pm \lambda_{n-1}$ which are different from 0 . Because $S$ is symmetric, it follows from the proposition that $\mathcal{X}\left(\mathbb{P}_{0}\left(M_{\varphi_{0}, 0}+E_{11}\right)\right)$ has purely imaginary spectrum, with two eigenvalues equal to 0 , and $n-1$ simple eigenvalues near $\mathbf{i}(1+\varphi)$ and another $n-1$ near $-\mathbf{i}(1+\varphi)$. Furthermore, since $E_{11}$ is proportional to $\varepsilon$, and $S$ has simple eigenvalues separated by $\mathcal{O}(1)$, we conclude

Corollary 5.4. The eigenvalues of $\mathcal{X}\left(\mathbb{P}_{0}\left(M_{\varphi_{0}, 0}+E_{11}\right)\right)$ are purely imaginary and satisfy $\left|\mu_{i}-\mu_{j}\right|>C_{n}^{\prime} \varepsilon$ when $i \neq j$. The constant $C_{n}^{\prime}>0$ only depends on $n$. The eigenfunctions, in the $\mathcal{X}(\cdot)$ basis, have non-zero component at position $2+n-1$ and $2 n$. 
We will now show that the remaining terms of $\mathcal{X}\left(\mathbb{P} M_{\varphi, \varepsilon}\right)$ only give rise to corrections of order $\mathcal{O}_{2}$, both in the spectrum and in the eigendirections (on the subspace spanned by $\left.X^{*}(\xi, \eta)^{\top}\right)$. For the terms which are of order $\mathcal{O}_{2}$, there is nothing to prove, since they perturb a matrix whose spectrum is separated by $\mathcal{O}(\varepsilon)$.

But there are terms of order $\mathcal{O}(\varepsilon)$. They are of the form $\mathcal{X}\left(\mathbb{P}_{2} M_{0,0}\right)$ or $\mathcal{X}\left(\mathbb{P}_{0} E_{12}\right)$. Here, the special form of the matrices comes into play, and the following lemma formulates the key point:

Lemma 5.5. Let $U, V$ be $(r+s) \times(r+s)$ matrices of the form

$$
U=\left(\begin{array}{l|l}
U_{1} & \\
\hline & U_{2}
\end{array}\right), \quad V=\left(\begin{array}{l|l} 
& V_{1} \\
\hline V_{2} &
\end{array}\right),
$$

where $U_{1}$ is an $r \times r$ square matrix, $U_{2}$ an $s \times s$ square matrix and $V_{1}$ and $V_{2}$ are $s \times r$ and $r \times s$ matrices, respectively. Let $x=\left(0, x_{2}\right)^{\top}$ be an eigenvector of $U$ (here $\left.x_{2} \in \mathbb{R}^{s}\right)$. Then

$$
\langle x \mid V x\rangle=0 .
$$

Proof. Obvious.

We apply this lemma to the two matrices $\mathcal{X}\left(\mathbb{P}_{2} M_{0,0}\right)$ and $\mathcal{X}\left(\mathbb{P}_{0} E_{12}\right)$, which play the role of $V$ in the lemma. From Proposition 5.3 we conclude that the eigenvectors of Eq. (5.2) are of the form

$$
x_{1}=(0,0, *, \ldots, *, 0, \ldots, 0)^{\top} \text { or } x_{2}=(0,0,0, \ldots, 0, *, \ldots, *)^{\top} .
$$

Therefore Eq. (5.3) applies in this case, and thus the first order contributions of $\mathcal{X}\left(\mathbb{P}_{2}\right)$ resp. $\mathcal{X}\left(E_{12}\right)$ vanish. Thus, as the spectra are $\varepsilon$-separated and simple by Corollary 5.4, we see that for small enough $|\varepsilon|$, the spectrum maintains the splitting properties when the second order perturbations (in $\varepsilon$ ) are added. Note that, since $\mathcal{X}\left(M_{\varphi_{0}, 0}\right)=(1+$ $\left.\varphi_{0}\right) \mathcal{X}\left(M_{0,0}\right)$ by Eq. (4.5) and as $\mathcal{X}\left(M_{0,0}\right)$ has the form Eq. (4.4), the effect of $\varphi_{0}$ is to just shift the spectrum globally, without changing the spacing of the eigenvalues within a block. This completes the proof of Theorem 5.1.

We end the section with the

Proof of Proposition 5.3. Suppose $x=\left(x_{1}, \ldots, x_{n}\right)^{\top}$ is an eigenfunction with eigenvalue $\lambda$. Then, from the form of $U$, we have

$$
\begin{aligned}
\left(U_{11} x_{1}+U_{12} x_{2}\right) & =\lambda x_{1}, \text { so that } x_{2}=\frac{1}{U_{12}}\left(\lambda-U_{11}\right) x_{1}, \\
\left(U_{21} x_{1}+U_{22} x_{2}+U_{23} x_{3}\right) & =\lambda x_{2}, \text { so that } \\
x_{3} & =\frac{1}{U_{23}}\left(\left(\lambda-U_{22}\right) x_{2}-U_{21} x_{1}\right) \\
& =\frac{1}{U_{23}}\left(\left(\lambda-U_{22}\right) \frac{1}{U_{12}}\left(\lambda-U_{11}\right)-U_{21}\right) x_{1} .
\end{aligned}
$$

Continuing in this way, we can write $x_{j}=C_{j} x_{1}$ for some constant $C_{j}$ defined in terms of the $U_{i j}$ and $\lambda$. But then, if $x_{1}=0$ all other $x_{j}$ are 0 , and we have not found a non-trivial eigenfunction. The same argument rules out the case $x_{n}=0$. Note that other $x_{j}$ can vanish.

The proof of 2 is by the same argument: If we normalize, say, $x_{1}=1$, the inductive steps above show that the eigenfunctions are uniquely determined by the eigenvalues. Hence, since there is a complete set of eigenvectors, all eigenvalues are simple. 


\section{The Effect of the Dissipation on the Semigroup}

In order to control the evolution of $\zeta(t)$, we need to understand precisely the bounds on the evolution of the semigroup generated by the linear part of the equation. We find that all modes in the subspace orthogonal to the zero eigendirections are uniformly contracted with a rate proportional to $\gamma \varepsilon$. This is somewhat surprising due to the localized nature of the dissipative term in the equation. However, if follows from the facts we have demonstrated above. Namely, we have shown in Theorem 5.1 that the eigenvectors in the $\mathcal{X}(\cdot)$ representation have nonzero last component, and are isolated, and so we conclude from standard perturbation theory that, adding dissipation $\Gamma$ moves these eigenvalues into the left half plane, by an amount proportional to $\gamma \varepsilon$ (up to higher order terms). We now check that the coefficients of the term proportional to $\gamma \varepsilon$ are all non-zero (and depend only on $n$ ).

Let

$$
\mathcal{L}_{\varphi_{0}, \gamma}=M_{\varphi_{0}, \varepsilon}-\Gamma, \quad \mathcal{X}\left(\mathcal{L}_{\varphi_{0}, \gamma}\right)=\mathcal{X}\left(M_{\varphi_{0}, \varepsilon}\right)-\mathcal{X}(\Gamma) .
$$

An explicit calculation shows that

$$
\mathcal{X}(\Gamma)=\left(\begin{array}{cc|cc|cc}
0 & 0 & & & & \\
0 & 0 & & & & \\
\hline & 0 & 0 & 0 & & \\
& 0 & 0 & 0 & & \\
& 0 & 0 & \gamma \varepsilon & & \\
\hline & & & 0 & 0 & 0 \\
& & & 0 & 0 & 0 \\
0 & & & 0 & \gamma \varepsilon
\end{array}\right) .
$$

Proposition 6.1. There is a constant $\gamma_{0}>0$, depending only on $n$ such that for $\gamma \in$ $\left[0, \gamma_{0}\right]$ one has the bound

$$
\left\|e^{t \mathcal{L}_{\varphi_{0}, \gamma}} X^{*} \zeta\right\| \leq\left(1+C_{n} \gamma\right) e^{-\varkappa_{n} \gamma \varepsilon t}\left\|X^{*} \zeta\right\|,
$$

for some $\varkappa_{n}>0$ and $C_{n}>0$, depending only on $n$.

Proof. This result follows from the classical perturbation theory for eigenvalues and eigenfunctions. By Theorem 5.1 we know that for $\gamma=0$ the purely imaginary eigenvalues are simple, and pairwise separated by $C \varepsilon$, with $C>0$ depending only on $n$. We focus on the eigenvalues close to $+\mathbf{i}-$ those near $-\mathbf{i}$ are handled by an entirely analogous procedure. From Prop.5.3 we see that the eigenvectors $v_{j}, j=1, \ldots, n-1$ corresponding to these eigenvalues have a non-vanishing last component, and therefore there exists some $C^{\prime}>0$, depending only on $n$, such that $\left\langle v_{j}, \Gamma v_{j}\right\rangle>C^{\prime} \gamma \varepsilon$, Therefore, up to higher order terms, standard perturbation theory for simple eigenvalues tells us that the spectrum of $\mathcal{X}\left(\mathcal{L}_{\varphi, \gamma}\right)$ has (twice) $n-1$ eigenvalues in the negative halfplane at a distance of $\mathcal{O}(\gamma \varepsilon)$ from the imaginary axis.

We next show that the eigendirections make an angle of at most $\mathcal{O}(\gamma)$ from the orthogonality of the eigendirections of the symmetric matrix $\mathcal{X}\left(M_{\varphi, \varepsilon}\right)$, thus proving the bound Eq. (6.3). From perturbation theory (see e.g., [12][I.\$5.3]), the projection onto one of these eigenspaces is given by

$$
P_{j}=-\frac{1}{2 \pi \mathbf{i}} \int_{C_{j}} R(z) \mathrm{d} z,
$$


where the contour $C_{j}$ is a circle of radius $\mathcal{O}(\varepsilon)$ around the eigenvalue of the problem for $\gamma=0$ and $R$ is the resolvent. The perturbed eigenvalue (which moves a distance $\sim-K_{j} \gamma \varepsilon$ ) lies inside this circle, if $\gamma<\gamma_{0}$ is sufficiently small, where $\gamma_{0}$ depends only on $n$ but not on $\varepsilon$, so long as $|\varepsilon|<\varepsilon_{0}$, for some fixed $\varepsilon_{0}$. Therefore, $\left\|P_{j}\right\|<1+\mathcal{O}(\gamma)$, since the contour integral over the circle leads to a bound $1 / \varepsilon$ which cancels the factor $\varepsilon$ in $\gamma \varepsilon$.

Note that since the change of variables matrix $X$ is orthogonal, these decay estimates also hold in the original coordinates, i.e.,

Corollary 6.2. There is a constant $C_{n}$ such that

$$
\left\|e^{t \mathcal{L}_{\varphi_{0}, \gamma}} \zeta\right\| \leq\left(1+C_{n} \gamma\right) e^{-\varkappa_{n} \gamma \varepsilon t}\|\zeta\|
$$

\section{Projecting onto the Complement of the 0 Eigenspace}

In this section, we reexamine equations (3.3)-(3.5) to derive carefully, and explicitly, the equations for the evolution of the variables $\varphi, \vartheta$, and $\zeta$. In particular, we look at the constraints on these equations imposed by the requirement that $\zeta$ remains in the range of $\mathbb{P}=\mathbb{P}_{\varphi_{0}}$. As $\varphi$ changes with time, the projection will also generate terms involving $\varphi(t)-\varphi_{0}$. We will bound these terms carefully, since they lead to secular growth in $\zeta$.

As we will often have to compare $p(\varphi(t))$ to $p\left(\varphi_{0}\right)$, it is useful to bound this difference as $\mathcal{O}(\delta)$ with

$$
\delta=\varphi(t)-\varphi_{0}
$$

We will only be interested in small $\delta$.

We fix a $\varphi_{0}$ small enough for Theorem 5.1 to apply. We next analyze the terms on the r.h.s of Eq. (3.5), one by one, using that $\zeta$ is orthogonal to the $n_{\varphi_{0}}^{(j)}$.

Lemma 7.1. Consider the linear evolution operator

$$
U=\left(\begin{array}{cc}
0 & -\left((L-\varphi)+(p(\varphi))^{2}\right) \\
(L-\varphi)+3(p(\varphi))^{2} & 0
\end{array}\right)
$$

Then,

$$
\begin{aligned}
\left\langle n_{\varphi_{0}}^{(2)} \mid U \zeta\right\rangle & =\mathcal{O}(\delta)\|\zeta\|, \\
\left\langle n_{\varphi_{0}}^{(1)} \mid U \zeta\right\rangle & =\mathcal{O}(\delta)\|\zeta\|, \\
\mathbb{P}_{\varphi_{0}} U \zeta & =U \zeta+\mathcal{O}(\delta)\|\zeta\| .
\end{aligned}
$$

Proof. Note that

$$
\left\langle n_{\varphi_{0}}^{(2)} \mid U \zeta\right\rangle=\left\langle U^{*} n_{\varphi_{0}}^{(2)} \mid \zeta\right\rangle
$$

and so,

$$
U^{*} n_{\varphi_{0}}^{(2)}=\left(\begin{array}{cc}
0 & \left((L-\varphi)+3(p(\varphi))^{2}\right) \\
-\left(L-\varphi+(p(\varphi))^{2}\right) & 0
\end{array}\right)\left(\begin{array}{c}
p\left(\varphi_{0}\right) \\
0
\end{array}\right)=\left(\begin{array}{c}
\mathcal{O}(\delta) \\
0
\end{array}\right)
$$


We use here, and throughout, the smoothness of $p(\varphi)$ and the expansion of $p(\varphi)$. The replacement of $\varphi$ by $\varphi_{0}$ therefore leads to an error term in Eq. (7.1) of the form

$$
\mathcal{O}(\delta)\|\xi\| \leq \mathcal{O}(\delta)\|\zeta\|
$$

This proves Eq. (7.1).

We next study $\left\langle n_{\varphi_{0}}^{(1)} \mid U \zeta\right\rangle$ and take the adjoint $\left\langle U^{*} n_{\varphi_{0}}^{(1)} \mid \zeta\right\rangle$, using $n_{\varphi_{0}}^{(1)}=(2+\mathcal{O}(\varepsilon+$ $\left.\left.\varphi_{0}\right)\right)\left(0, \partial_{\varphi_{0}} p\left(\varphi_{0}\right)\right)^{\top}$. Recall that

$$
\left(\begin{array}{cc}
0 & B_{\varphi_{0}} \\
A_{\varphi_{0}} & 0
\end{array}\right) n_{\varphi_{0}}^{(1)}=n_{\varphi_{0}}^{(2)}
$$

which is orthogonal to $\zeta=(\xi, \eta)^{\top}$. We write

$$
U^{*}=\left(\begin{array}{cc}
0 & B_{\varphi}-B_{\varphi_{0}} \\
A_{\varphi}-A_{\varphi_{0}} & 0
\end{array}\right)+\left(\begin{array}{cc}
0 & B_{\varphi_{0}} \\
A_{\varphi_{0}} & 0
\end{array}\right),
$$

and therefore

$$
\begin{aligned}
\left\langle n_{\varphi_{0}}^{(1)} \mid U \zeta\right\rangle & =\left\langle\left(\begin{array}{cc}
0 & B_{\varphi}-B_{\varphi_{0}} \\
A_{\varphi}-A_{\varphi_{0}} & 0
\end{array}\right) n_{\varphi_{0}}^{(1)} \mid \zeta\right\rangle \quad+\left\langle\left(\begin{array}{cc}
0 & B_{\varphi_{0}} \\
A_{\varphi_{0}} & 0
\end{array}\right) n_{\varphi_{0}}^{(1)} \mid \zeta\right\rangle \\
& =\mathcal{O}(\delta)\|\zeta\|,
\end{aligned}
$$

since the second term is zero by construction. This proves Eq. (7.2). The identity Eq. (7.3) follows.

Lemma 7.2. The $\Gamma$-dependent terms of Eq. (3.5) lead to the bounds

$$
\begin{gathered}
\left\langle n_{\varphi_{0}}^{(2)} \mid\left(\begin{array}{c}
-C_{\Gamma} \xi-C_{\Gamma} p(\varphi) \\
-C_{\Gamma} \eta
\end{array}\right)\right\rangle=\mathcal{O}\left(\gamma \varepsilon^{n}\right)\|\zeta\|-2(1+\mathcal{O}(\delta)) \gamma \varepsilon^{2 n-1}, \\
\left\langle n_{\varphi_{0}}^{(1)} \mid\left(\begin{array}{c}
-C_{\Gamma} \xi-C_{\Gamma} p(\varphi) \\
-C_{\Gamma} \eta
\end{array}\right)\right\rangle=\mathcal{O}\left(\gamma \varepsilon^{n}\right)\|\zeta\|, \\
\left\|\mathbb{P}_{\varphi_{0}} \Gamma \zeta-\Gamma \zeta\right\| \leq C \gamma \varepsilon^{n}\|\zeta\| .
\end{gathered}
$$

Proof. From the definition of $n_{\varphi_{0}}^{(2)}$ we get

$$
\begin{aligned}
& 2\left\langle\left(\begin{array}{c}
p\left(\varphi_{0}\right) \\
0
\end{array}\right) \mid\left(\begin{array}{c}
-C_{\Gamma} \xi-C_{\Gamma} p(\varphi) \\
-C_{\Gamma} \eta
\end{array}\right)\right\rangle \\
& =-2 \gamma \varepsilon \xi_{n} p\left(\varphi_{0}\right)_{n}-2 \gamma \varepsilon\left(p\left(\varphi_{0}\right)\right)_{n}(p(\varphi))_{n} \\
& =\mathcal{O}(1) \gamma \varepsilon \xi_{n} \varepsilon^{n-1}-2 \gamma \varepsilon^{2 n-1}(1+\mathcal{O}(\delta)),
\end{aligned}
$$

using the expansion of $p(\varphi)$ in powers of $\varepsilon$, and observing that $C_{\Gamma}$ is proportional to $\gamma \varepsilon$.

Similarly, from the definition of $n_{\varphi_{0}}^{(1)}$, we get

$$
\left\langle n_{\varphi_{0}}^{(1)} \mid\left(\begin{array}{c}
C_{\Gamma} \xi+C_{\Gamma} p(\varphi) \\
C_{\Gamma} \eta
\end{array}\right)\right\rangle=\partial_{\varphi_{0}} p\left(\varphi_{0}\right) \cdot C_{\Gamma} \eta=\gamma \varepsilon\left(n_{\varphi_{0}}^{(1)}\right)_{n} \eta_{n}=\mathcal{O}(1) \gamma \varepsilon^{n} \eta_{n},
$$

using the expansion for $\left(n_{\varphi_{0}}^{(1)}\right)_{j}=\partial_{\varphi_{0}} p_{j}\left(\varphi_{0}\right)=\mathcal{O}\left(\varepsilon^{j-1}\right)$. The last equation follows from the first two. 
Lemma 7.3. Consider the terms involving $(t \dot{\varphi}+\dot{\vartheta})$. We have, omitting throughout the factor $(t \dot{\varphi}+\dot{\vartheta})$ :

$$
\begin{aligned}
\left\langle n_{\varphi_{0}}^{(2)} \mid\left(\begin{array}{c}
\eta \\
-\xi-p(\varphi)
\end{array}\right)\right\rangle & =(2+\mathcal{O}(\varepsilon+\varphi))\|\zeta\|, \\
\left\langle n_{\varphi_{0}}^{(1)} \mid\left(\begin{array}{c}
\eta \\
-\xi-p(\varphi)
\end{array}\right)\right\rangle & =-1+\mathcal{O}(\delta+\varepsilon)+\mathcal{O}(1)\|\zeta\|, \\
\mathbb{P}_{\varphi_{0}}\left(\begin{array}{c}
\eta \\
-\xi-p(\varphi)
\end{array}\right) & =\left(\begin{array}{c}
\mathcal{O}(\|\zeta\|) \\
\mathcal{O}(\delta+\varepsilon+\|\zeta\|)
\end{array}\right) .
\end{aligned}
$$

Proof. Equation (7.7) follows by observing that

$$
\left\langle\left(\begin{array}{c}
p\left(\varphi_{0}\right) \\
0
\end{array}\right) \mid\left(\begin{array}{c}
\eta \\
-\xi
\end{array}\right)\right\rangle=\left(p\left(\varphi_{0}\right) \cdot \eta\right),
$$

and

$$
\left\langle\left(\begin{array}{c}
p\left(\varphi_{0}\right) \\
0
\end{array}\right) \mid\left(\begin{array}{c}
0 \\
-p(\varphi)
\end{array}\right)\right\rangle=0,
$$

and using $\left\|p\left(\varphi_{0}\right)\right\|=1+\mathcal{O}(\varepsilon+\varphi)$. To prove Eq. (7.8), observe that, by our normalization,

$$
\left\langle n_{\varphi_{0}}^{(1)} \mid\left(\begin{array}{c}
0 \\
p\left(\varphi_{0}\right)
\end{array}\right)\right\rangle=\left\langle n_{\varphi_{0}}^{(1)} \mid v_{\varphi_{0}}^{(1)}\right\rangle=1,
$$

and therefore,

$$
\left\langle n_{\varphi_{0}}^{(1)} \mid\left(\begin{array}{c}
0 \\
-p(\varphi)
\end{array}\right)\right\rangle=-1+\mathcal{O}(\delta) .
$$

On the other hand,

$$
\left\langle n_{\varphi_{0}}^{(1)} \mid\left(\begin{array}{c}
\eta \\
-\xi
\end{array}\right)\right\rangle=-\partial_{\varphi_{0}} p\left(\varphi_{0}\right) \xi
$$

and thus Eq. (7.8) follows. Finally Eq. (7.9) follows from Eqs. (7.7) and (7.8);

$$
\begin{aligned}
\mathbb{P}_{\varphi_{0}}\left(\begin{array}{c}
\eta \\
-\xi-p(\varphi)
\end{array}\right)= & \left(\begin{array}{c}
\eta \\
-\xi-p(\varphi)
\end{array}\right) \\
& -(2+\mathcal{O}(\varepsilon))\left(\begin{array}{c}
\partial_{\varphi_{0}} p\left(\varphi_{0}\right) \\
0
\end{array}\right)\left(p\left(\varphi_{0}\right) \cdot \eta\right) \\
& -\left(\begin{array}{c}
0 \\
p\left(\varphi_{0}\right)
\end{array}\right)(-1+\mathcal{O}(\delta+\varepsilon+\|\zeta\|)) .
\end{aligned}
$$

The term involving $\eta$ cancels by the normalization of $v^{(2)}$ and $n^{(2)}$. The term $-p\left(\varphi_{0}\right)$. $(-1)$ cancels with $-p(\varphi)$ up to $\mathcal{O}(\delta+\varepsilon)$, and thus, Eq. (7.9) follows. 
Lemma 7.4. The terms involving $\dot{\varphi}$ are bounded as follows (omitting the factor $\dot{\varphi}$ ):

$$
\begin{aligned}
\left\langle n_{\varphi_{0}}^{(2)} \mid\left(\begin{array}{c}
-\partial_{\varphi} p(\varphi) \\
0
\end{array}\right)\right\rangle & =-1+\mathcal{O}(\delta), \\
\left\langle n_{\varphi_{0}}^{(1)} \mid\left(\begin{array}{c}
-\partial_{\varphi} p(\varphi) \\
0
\end{array}\right)\right\rangle & =0, \\
\mathbb{P}_{\varphi_{0}}\left(\begin{array}{c}
-\partial_{\varphi} p(\varphi) \\
0
\end{array}\right) & =\left(\begin{array}{c}
\mathcal{O}(\delta) \\
0
\end{array}\right) .
\end{aligned}
$$

Proof. Recall that

$$
\left(\begin{array}{c}
\partial_{\varphi} p(\varphi) \\
0
\end{array}\right) \sim(1 / 2,0, \ldots, 0)^{\top}
$$

and so, since $n_{\varphi_{0}}^{(2)} \sim 2\left(p\left(\varphi_{0}\right), 0\right)^{\top}$, we find

$$
\begin{aligned}
\left\langle n_{\varphi_{0}}^{(2)} \mid\left(\begin{array}{c}
-\partial_{\varphi} p(\varphi) \\
0
\end{array}\right)\right\rangle & =\left\langle 2\left(\begin{array}{c}
p\left(\varphi_{0}\right) \\
0
\end{array}\right) \mid\left(\begin{array}{c}
-\partial_{\varphi} p(\varphi) \\
0
\end{array}\right)\right\rangle \\
& =-2 p\left(\varphi_{0}\right) \cdot \partial_{\varphi} p(\varphi)=-1+\mathcal{O}(\delta),
\end{aligned}
$$

which is Eq. (7.10). From the form of $n^{(1)}$, Eq. (7.11) is obvious. Finally,

$$
\mathbb{P}_{\varphi_{0}}\left(\begin{array}{c}
-\partial_{\varphi} p(\varphi) \\
0
\end{array}\right)=\left(\begin{array}{c}
-\partial_{\varphi} p(\varphi) \\
0
\end{array}\right)+\left(\begin{array}{c}
\partial_{\varphi_{0}} p\left(\varphi_{0}\right) \\
0
\end{array}\right)+\left(\begin{array}{c}
\mathcal{O}(\delta) \\
0
\end{array}\right)
$$

We now combine the Lemmas 7.1-7.4. Note that $\mathbb{Q}_{\varphi_{0}} \equiv \mathbb{1}-\mathbb{P}_{\varphi_{0}}$ projects on a two-dimensional space. Let $\mathbb{Q}^{(j)}=\left|v_{\varphi_{0}}^{(j)}\right\rangle\left\langle n_{\varphi_{0}}^{(j)}\right|, j=1,2$.

For $j=2$, we get contributions:

From Eq. (7.1), we have $\mathbb{Q}^{(2)} U \zeta=\mathcal{O}(\delta)\|\zeta\|$.

From Eq. (7.4) we get $\mathbb{Q}^{(2)}\left(\begin{array}{c}C_{\Gamma}(\xi+p(\varphi)) \\ C_{\Gamma} \eta\end{array}\right)=-2(1+\mathcal{O}(\delta)) \gamma \varepsilon^{2 n-1}+\mathcal{O}\left(\gamma \varepsilon^{n}\right)\|\zeta\|$.

From Eq. (7.7) we get $(t \dot{\varphi}+\dot{\vartheta}) \mathbb{Q}^{(2)}\left(\begin{array}{c}\eta \\ -\xi-p(\varphi)\end{array}\right)=(t \dot{\varphi}+\dot{\vartheta}) \mathcal{O}(\|\zeta\|)$, and

from Eq. (7.10) we get $\dot{\varphi} \mathbb{Q}^{(2)}\left(\begin{array}{c}-\partial_{\varphi} p(\varphi) \\ 0\end{array}\right)=\dot{\varphi}(-1+\mathcal{O}(\delta))$.

Similarly, for $j=1$, we get contributions:

From Eq. (7.2), we have $\mathbb{Q}^{(1)} U \zeta=\mathcal{O}(\delta)\|\zeta\|$.

From Eq. (7.5) we get $\mathbb{Q}^{(1)}\left(\begin{array}{c}C_{\Gamma}(\xi+p(\varphi)) \\ C_{\Gamma} \eta\end{array}\right)=\mathcal{O}\left(\gamma \varepsilon^{n}\right)\|\zeta\|$.

From Eq. (7.8) we get

$(t \dot{\varphi}+\dot{\vartheta}) \mathbb{Q}^{(1)}\left(\begin{array}{c}\eta \\ -\xi-p(\varphi)\end{array}\right)=(t \dot{\varphi}+\dot{\vartheta})(-1+\mathcal{O}(\delta+\varepsilon+\|\zeta\|)$, and

from Eq. (7.11) we get $\dot{\varphi} \mathbb{Q}^{(1)}\left(\begin{array}{c}-\partial_{\varphi} p(\varphi) \\ 0\end{array}\right)=0$.

By construction we have that $\mathbb{Q}_{\varphi_{0}} \equiv \mathbb{1}-\mathbb{P}_{\varphi_{0}}$ projects on the null-space, and therefore

$$
\mathbb{Q}_{\varphi_{0}} \dot{\zeta}=0
$$


Since $\mathbb{Q}_{\varphi_{0}} \dot{\zeta}=0$, we find, upon summing, for the "1" component (and recalling the nonlinear terms in (3.5)), we find

$$
\begin{aligned}
0= & \mathcal{O}(\delta)\|\zeta\|+(-2+\mathcal{O}(\delta)) \gamma \varepsilon^{2 n-1} \\
& +\mathcal{O}\left(\gamma \varepsilon^{n}\|\zeta\|\right)+(t \dot{\varphi}+\dot{\vartheta}) \mathcal{O}(\|\zeta\|)+\dot{\varphi}(-1+\mathcal{O}(\delta))+\mathcal{O}\left(\|\zeta\|^{2}\right),
\end{aligned}
$$

and for the "2" component:

$$
0=\left(\mathcal{O}(\delta)+\mathcal{O}\left(\gamma \varepsilon^{n}\right)\|\zeta\|-(t \dot{\varphi}+\dot{\vartheta})(1+\mathcal{O}(\delta+\varepsilon+\|\zeta\|))+\mathcal{O}\left(\|\zeta\|^{2}\right) .\right.
$$

Finally, using the projection $\mathbb{P}_{\varphi_{0}}$, we find:

From Eq. (7.3), $\mathbb{P}_{\varphi_{0}} U \zeta=U \zeta+\mathcal{O}(\delta)\|\zeta\|$,

from Eq. (7.6), $\mathbb{P}_{\varphi_{0}} \Gamma \zeta=\Gamma \zeta+\mathcal{O}\left(\gamma \varepsilon^{n}\right)\|\zeta\|$,

from Eq. (7.9), $(t \dot{\varphi}+\dot{\vartheta}) \mathbb{P}_{\varphi_{0}}\left(\begin{array}{c}\eta \\ -\xi-p(\varphi)\end{array}\right)=(t \dot{\varphi}+\dot{\vartheta})\left(\begin{array}{c}0 \\ \mathcal{O}(\delta+\varepsilon)\end{array}\right)$,

and finally from Eq. (7.12), $\dot{\varphi} \mathbb{P}_{\varphi_{0}}\left(\begin{array}{c}-\partial_{\varphi} p(\varphi) \\ 0\end{array}\right)=\dot{\varphi}\left(\begin{array}{c}\mathcal{O}(\delta) \\ 0\end{array}\right)$.

Summing these terms, we get

$$
\dot{\zeta}=\mathcal{L}_{\varphi_{0}, \gamma} \zeta+(t \dot{\varphi}+\dot{\vartheta})\left(\begin{array}{c}
0 \\
\mathcal{O}(\delta+\varepsilon)
\end{array}\right)+\dot{\varphi}\left(\begin{array}{c}
\mathcal{O}(\delta) \\
0
\end{array}\right)+\mathcal{O}\left(\|\zeta\|^{2}\right) .
$$

Simplifying the notation somewhat, and substituting Eq. (7.14) into Eq. (7.13) we formulate Eqs. (7.13)-(7.15) as a proposition:

\section{Proposition 7.5. One has}

$$
\begin{aligned}
t \dot{\varphi}+\dot{\vartheta}= & \mathcal{O}\left(\delta+\gamma \varepsilon^{n}\right)\|\zeta\|+\mathcal{O}\left(\|\zeta\|^{2}\right) \\
\dot{\varphi}= & -(2+\mathcal{O}(\delta)) \gamma \varepsilon^{2 n-1}+\mathcal{O}\left(\delta+\gamma \varepsilon^{n}\right)\|\zeta\|+\mathcal{O}\left(\|\zeta\|^{2}\right) \\
\dot{\zeta}= & \mathcal{L}_{\varphi_{0}, \gamma} \zeta+\mathcal{O}\left(\delta+\gamma \varepsilon^{n}\right)\|\zeta\|\left(\begin{array}{c}
0 \\
\mathcal{O}(\delta+\varepsilon)
\end{array}\right) \\
& -\left(\begin{array}{c}
(2+\mathcal{O}(\delta)) \gamma \varepsilon^{2 n-1} \mathcal{O}(\delta) \\
0
\end{array}\right)+\mathcal{O}\left(\|\zeta\|^{2}\right) .
\end{aligned}
$$

Here, as $\zeta=(\xi, \eta)^{\top}$,

$$
\mathcal{L}_{\varphi_{0}, \gamma} \zeta=\left(\begin{array}{cc}
0 & A_{\varphi_{0}} \\
B_{\varphi_{0}} & 0
\end{array}\right)\left(\begin{array}{l}
\xi \\
\eta
\end{array}\right)-\left(\begin{array}{cc}
C_{\Gamma} & 0 \\
0 & C_{\Gamma}
\end{array}\right)\left(\begin{array}{l}
\xi \\
\eta
\end{array}\right)
$$

\section{Bounds on the Evolution of $\zeta$}

In principle, $\|\zeta\|$ can grow as the system evolves, and there are two possible causes. First, for short times, the bound Eq. (6.4)

$$
\left\|e^{t \mathcal{L}_{\varphi_{0}, \gamma}} \zeta\right\| \leq\left(1+C_{n} \gamma\right) e^{-\varkappa_{n} \gamma \varepsilon t}\|\zeta\|,
$$

does not contract. Secondly, $\zeta(t)$ is orthogonal to the cylinder of breathers at the initial point $\left(\varphi_{0}, \vartheta_{0}\right)$, but as $\varphi$ and $\vartheta$ evolve with time, this is no longer the case. We must periodically reorthogonalize $\zeta(t)$ by a procedure which we detail in the next section, 
and which replaces $\zeta(T)$ by $\widehat{\zeta}$. As we will show in Eq. (9.4), this leads to a growth which is bounded by

$$
\|\widehat{\zeta}\| \leq\left(1+C_{R}\left(\varphi(T)-\varphi_{0}\right) \gamma \varepsilon^{n}\right)\|\zeta(T)\| .
$$

In this section we show that the contraction in the semigroup generated by the dissipative terms in the equation is sufficient to overcome those growths, if we wait a sufficiently long time. We will show (up to details spelled out below) that if $\|\zeta(0)\| \leq \gamma \varepsilon^{n}$, and $T=C_{T} / \varepsilon$, then $\left(1+C_{R}\left|\varphi(T)-\varphi_{0}\right| \gamma \varepsilon^{n}\right)\|\zeta(T)\| \leq \gamma \varepsilon^{n}$. Furthermore for all $t \in[0, T]$ one has $\|\zeta(t)\| \leq 2 \gamma \varepsilon^{n}$. To prove such statements, we reconsider the equations of Proposition 7.5 which we rewrite in a slightly simplified way: We define

$$
\delta(t)=\varphi(t)-\varphi_{0},
$$

and then

$$
\begin{aligned}
& \dot{s}=\mathcal{O}\left(\delta+\gamma \varepsilon^{n}\right)\|\zeta\|+\mathcal{O}\left(\|\zeta(t)\|^{2}\right) \\
& \dot{\delta}=-(2+\mathcal{O}(\delta)) \gamma \varepsilon^{2 n-1}+\mathcal{O}\left(\delta+\gamma \varepsilon^{n}\right)\|\zeta\|+\mathcal{O}\left(\|\zeta(t)\|^{2}\right), \\
& \dot{\zeta}=\mathcal{L}_{\varphi_{0}, \gamma} \zeta+\dot{s}\left(\begin{array}{c}
0 \\
\mathcal{O}(\delta+\varepsilon)
\end{array}\right)+\dot{\delta}\left(\begin{array}{c}
\mathcal{O}(\delta) \\
0
\end{array}\right)+\mathcal{O}\left(\|\zeta(t)\|^{2}\right) .
\end{aligned}
$$

Here, as $\zeta=(\xi, \eta)^{\top}$,

$$
\mathcal{L}_{\varphi_{0}, \gamma} \zeta=\left(\begin{array}{cc}
0 & A_{\varphi_{0}} \\
B_{\varphi_{0}} & 0
\end{array}\right)\left(\begin{array}{l}
\xi \\
\eta
\end{array}\right)-\left(\begin{array}{cc}
C_{\Gamma} & 0 \\
0 & C_{\Gamma}
\end{array}\right)\left(\begin{array}{l}
\xi \\
\eta
\end{array}\right) .
$$

Definition 8.1. We define the arrival time $T$ by

$$
T=\frac{8 C_{n}}{\varkappa_{n} \varepsilon} \equiv C_{T} \varepsilon^{-1}
$$

This definition ensures that

$$
\left(1+\frac{3}{2} C_{n} \gamma\right) e^{-\varkappa_{n} \gamma \varepsilon T / 4} \leq 1
$$

The remaining factor $e^{-\chi_{n} \gamma \varepsilon T / 4}$ will be used to bound $\left(1+C_{R}|\delta| \gamma \varepsilon^{n}\right)$, while another $e^{-x_{n} \gamma \varepsilon T / 2}$ will be used to bound the contributions from the mixed terms in Eqs. (8.2)(8.4).

Since we have a coupled system, we introduce a norm over times in $[0, T]$. Let $x=$ $(s, \delta, \zeta)$, and consider a family of functions

$$
\{x\}_{T}=\{x(\tau)\}_{\tau \in[0, T]} .
$$

We define

$$
\left\|\left|\{x\}_{t}\right|\right\|=\max \left(\sup _{\tau \in[0, t]}|s(\tau)|, \sup _{\tau \in[0, t]}|\delta(\tau)|, C_{\zeta}\|\zeta(\tau)\|\right) \text {, with } C_{\zeta}^{-1}=\gamma \varepsilon^{n} .
$$

The equations Eqs. (8.2)-(8.4) define an evolution $t \mapsto \mathcal{F}^{t}$. 
Theorem 8.2. Let $t \mapsto x(t)$ be a family of functions (not necessarily a solution of the system above) which satisfies $\left\|\{x\}_{T}\right\| \leq 2$. Define $\mathcal{F} x=\left\{\mathcal{F}^{\tau} x\right\}_{\tau \in[0, T]}$ as the family evolving from $x(0)$. If $\left\|\{x\}_{0}\right\| \mid \leq 2$ and $s(0)=\delta(0)=0$, then the solution of the system above satisfies

$$
\left\|\{\mathcal{F} x\}_{t}\right\| \mid \leq 4
$$

for all $t \in[0, T]$. In other words, $\mathcal{F}$ maps such initial conditions into the sphere of radius 4 .

Furthermore, when $\left\|\{x\}_{0}\right\| \leq 1$ (this means in particular $\|\zeta(0)\| \leq \gamma \varepsilon^{n}$ ) then the solution of the above system satisfies at time $T^{5}$ :

$$
\begin{aligned}
\mid \delta(T) & +2 \gamma \varepsilon^{2 n-1} T \mid \leq 2 \gamma \varepsilon^{2 n-1 / 2} T \\
\|\zeta(T)\| & \leq e^{-\varkappa_{n} \gamma \varepsilon T / 4} e^{-\varkappa_{n} \gamma \varepsilon T / 2}(1-\gamma \varepsilon)^{-1}\left(1+\frac{3}{2} C_{n} \gamma\right) \gamma \varepsilon^{n} \\
& \leq e^{-\varkappa_{n} \gamma \varepsilon T / 4} \gamma \varepsilon^{n}
\end{aligned}
$$

Corollary 8.3. Referring to Eq. (8.1) (i.e., Eq. (9.4)), we get the bound

$$
\begin{aligned}
\|\widehat{\zeta}\| & \leq\left(1+C_{R}\left(\varphi(T)-\varphi_{0}\right) \gamma \varepsilon^{n}\right)\|\zeta(T)\| \\
& \leq e^{-\varkappa_{n} \gamma \varepsilon T / 4}\left(1+C_{R}\left(\varphi(T)-\varphi_{0}\right) \gamma \varepsilon^{n}\right) \gamma \varepsilon^{n} \leq \gamma \varepsilon^{n} .
\end{aligned}
$$

In other words, $\|\widehat{\zeta}\|$ (at time $T$ ) stays within the region $\gamma \varepsilon^{n}$.

Remark 8.4. The norm $\||\cdot| \mid$ was introduced to allow for an a priori bound on $\zeta(t)$ which is needed because of our way to estimate the evolution of the coupled system Eqs. (8.2)(8.4).

Remark 8.5. We assumed $\delta(0)=0$ since that is the case which interests us. Also, by the gauge invariance, we may assume that $\vartheta_{0}=\vartheta(0)=0$.

Proof. We first study $\delta$.

Lemma 8.6. Assume $\left\|\left|\{x\}_{T}\right|\right\| \leq 2$. Then, we have, for $t \leq T=C_{T} / \varepsilon$,

$$
\left|\delta(t)+2 \gamma \varepsilon^{2 n-1} t\right| \leq 2 \gamma \varepsilon^{2 n-1 / 2} t .
$$

Remark 8.7. Note that this means that to lowest order in $\varepsilon, \delta(t) \sim 2 \gamma \varepsilon^{2 n-1} t$ for $0 \leq t \leq$ $T$, which is the rate we found in [1].

Proof of Lemma 8.6. It is here that we use the a priori bound, and later we will see that the actual orbit of $\zeta(\tau)$ indeed satisfies this bound. By the assumption, we have $\|\zeta(\tau)\| \leq 2 / C_{\zeta}=2 \gamma \varepsilon^{n}$ for $\tau \in[0, T]$. Therefore, we can bound $\delta(\tau)$ as follows: The Eq. (8.3) is of the form (with local names) and finite constants $C_{B}$ and $C_{C}$ :

$$
\begin{aligned}
\dot{\delta}(t) & =-A+B(t) \delta(t)+C(t), \\
A & =2 \gamma \varepsilon^{2 n-1}, \\
|B(t)| & \leq C_{B}\left(\gamma \varepsilon^{2 n-1}+\|\zeta(t)\|\right), \\
|C(t)| & \leq C_{C}\left(\gamma \varepsilon^{n}\|\zeta(t)\|+\|\zeta(t)\|^{2}\right) .
\end{aligned}
$$

We have $\delta(0)=0$. The equation for $u(t) \equiv \delta(t)+$ At reads

$$
\dot{u}=B(t) u(t)+(C(t)-A t \cdot B(t)) .
$$

\footnotetext{
5 We are not claiming that such bounds hold for all $t \leq T$. The effect of the dissipation needs time to set in (at least if we want to re-project onto a new axis after some time).
} 
Lemma 8.8. If $\left\|\{x\}_{T}\right\| \leq 2$, then

$$
|u(t)| \leq A t \varepsilon^{1 / 2} .
$$

This clearly proves Eq. (8.7) and hence Lemma 8.6.

Proof of Lemma 8.8. The solution of Eq. (8.8) is

$$
u(t)=\int_{0}^{t} \mathrm{~d} \tau(C(\tau)-A \tau B(\tau)) e^{\int_{\tau}^{t} \mathrm{~d} \tau^{\prime} B\left(\tau^{\prime}\right)} .
$$

Let $B_{\max }=\max _{\tau \in[0, t]}|B(\tau)|$, and $C_{\max }=\max _{\tau \in[0, t]}|C(\tau)|$. From the assumptions, we have, for sufficiently small $\varepsilon$,

$$
\begin{aligned}
B_{\max } t & \leq C_{T} C_{B} 2\left(\gamma \varepsilon^{2 n-1}+C_{\zeta}^{-1}\right) / \varepsilon \\
& \leq C_{T} C_{B} 2\left(\gamma \varepsilon^{2 n-2}+\varepsilon^{n-1 / 2}\right) \ll 2 \varepsilon, \\
C_{\max } & \leq C_{C} 2\left(\gamma \varepsilon^{n} C_{\zeta}^{-1}+4 C_{\zeta}^{-2}\right) \leq 4 C_{C} \gamma \varepsilon^{2 n},
\end{aligned}
$$

(where we have assumed that $\gamma<1 / 2$ ). The term coming from $C(\tau)$ in Eq. (8.9) is bounded by

$$
C_{\max } \frac{\left|1-e^{B_{\max } t}\right|}{B_{\max }} \leq 2 C_{\max } t,
$$

since $B_{\max } t \ll 1$.

This leads to a bound for $2 C_{\max } t$ of the form

$$
2 C_{\max } t \leq 8 C_{C} \gamma \varepsilon^{2 n} t,
$$

which is much smaller than $A t=2 \gamma \varepsilon^{2 n-1} t$ (when $\varepsilon$ is small enough). The term in the integral coming from $A t \cdot B(t)$ can be bounded as:

$$
\begin{gathered}
\int_{0}^{t} \mathrm{~d} \tau A \tau \cdot B_{\max } e^{(t-\tau) B_{\max }}=A \frac{\left|B_{\max } t-e^{B_{\max } t}+1\right|}{B_{\max }} \\
\leq A t\left(\frac{B_{\max } t}{2}+\mathcal{O}\left(\left(B_{\max } t\right)^{2}\right)\right) \leq A t B_{\max } t
\end{gathered}
$$

since we already showed $B_{\max } t \ll 2 \varepsilon$. Collecting terms, we get, for $t \leq C_{T} / \varepsilon$,

$$
|u(t)| \leq\left(8 C_{C} \gamma \varepsilon^{2 n}+2 \varepsilon A\right) t \leq A t \varepsilon^{1 / 2},
$$

which completes the proof of Lemma 8.8.

We continue the proof of Theorem 8.2. The evolution of $s$ is bounded in much the same way as that of $\delta$, and this is left to the reader. (We actually do not make use of these bounds.) We finally analyze the evolution of $\zeta$, Eq. (8.4), which controls the motion of the distance from the cylinder. By the estimates Eqs. (8.3) and (8.7) on $\delta$ and $\dot{\delta}$, we see that

$$
\mathcal{O}(\delta \dot{\delta})=\mathcal{O}\left(\gamma^{2} \varepsilon^{4 n-2} t\right)+\mathcal{O}\left(\gamma \varepsilon^{2 n-1} t+\gamma \varepsilon^{n}\right)\|\zeta\| .
$$

Therefore, the equation for $\dot{\zeta}$ takes the form

$$
\dot{\zeta}=\mathcal{L}_{\varphi, \gamma} \zeta+\mathcal{O}(\delta+\varepsilon) \cdot \mathcal{O}\left(\delta+\gamma \varepsilon^{n}\right)\|\zeta\|+\mathcal{O}\left(\|\zeta\|^{2}\right)+\mathcal{O}\left(\gamma^{2} \varepsilon^{4 n-2} t\right) .
$$


Using Eq. (8.7), this simplifies to

$$
\dot{\zeta}=\mathcal{L}_{\varphi, \gamma} \zeta+\mathcal{O}\left(\gamma \varepsilon^{n+1}+\gamma \varepsilon^{2 n-1} t\right)\|\zeta\|+\mathcal{O}\left(\|\zeta\|^{2}\right)+\mathcal{O}\left(\gamma^{2} \varepsilon^{4 n-2} t\right)
$$

From the estimates on the semigroup generated by $\mathcal{L}_{\varphi, \gamma}$ from Proposition 6.1 we conclude that

$$
\begin{aligned}
\|\zeta(t)\| \leq & \left(1+C_{n} \gamma\right) e^{-\varkappa_{n} \gamma \varepsilon t / 2}\left\|\zeta_{0}\right\|+R_{2}\left(1+C_{n} \gamma\right) \int_{0}^{t} e^{-\varkappa_{n} \gamma \varepsilon(t-s) / 2}\|\zeta(s)\|^{2} d s \\
& +\left(1+C_{n} \gamma\right) \int_{0}^{t} \mathrm{~d} \tau e^{-\varkappa_{n} \gamma \varepsilon \tau / 2} X
\end{aligned}
$$

where $X=\mathcal{O}\left(\gamma^{2} \varepsilon^{4 n-2}(t-\tau)\right)$ bounds the contribution from the last term in Eq. (8.10). We note that the contribution from $\mathcal{O}\left(\gamma \varepsilon^{n+1}+\gamma \varepsilon^{2 n-1} t\right)$ which also multiplies $\zeta$, has been absorbed into half the decay rate $\varkappa_{n} \gamma \varepsilon$.

Define

$$
Z(t)=\sup _{0 \leq \tau \leq t} e^{\varkappa_{n} \gamma \varepsilon \tau / 2}\|\zeta(\tau)\|
$$

Then, from (8.11), we see that

$$
\begin{aligned}
Z(t) \leq & \left(1+C_{n} \gamma\right)\left\|\zeta_{0}\right\| \\
& +R_{2}\left(1+C_{n} \gamma\right) \int_{0}^{t} e^{-\varkappa_{n} \gamma \varepsilon s} d s(Z(t))^{2}+C_{X}\left(\gamma^{2} \varepsilon^{4 n-2} t^{2} e^{\varkappa_{n} \gamma \varepsilon t / 2}\right) \\
\leq & \left(1+C_{n} \gamma\right)\left\|\zeta_{0}\right\|+\frac{2 R_{2}\left(1+C_{n} \gamma\right)}{\varkappa_{n} \gamma \varepsilon}\left(e^{\varkappa_{n} \gamma \varepsilon t / 2}-1\right)(Z(t))^{2} \\
& +C_{X}\left(\gamma^{2} \varepsilon^{4 n-2} t^{2} e^{\varkappa_{n} \gamma \varepsilon t / 2}\right) .
\end{aligned}
$$

Suppose that $\left\|\zeta_{0}\right\| \leq \gamma \varepsilon^{n}$. Then, by continuity, for $t$ small, we have

$$
\frac{2 R_{2}\left(1+C_{n} \gamma\right)}{\varkappa_{n} \gamma \varepsilon} Z(t) \leq \gamma \varepsilon \text {. }
$$

Define $T^{*}$ to be the largest value such that

$$
\sup _{0 \leq t \leq T^{*}} \frac{2 R_{2}\left(1+C_{n} \gamma\right)}{\varkappa_{n} \gamma \varepsilon} Z(t) \leq \gamma \varepsilon .
$$

Then,

$$
\left(1-\frac{2 R_{2}\left(1+C_{n} \gamma\right)}{\varkappa_{n} \gamma \varepsilon} Z(t)\right) Z(t) \leq\left(1+C_{n} \gamma\right)\left\|\zeta_{0}\right\|+C_{X}\left(\gamma^{2} \varepsilon^{4 n-2} t^{2} e^{\varkappa_{n} \gamma \varepsilon t / 2}\right),
$$

or

$$
Z(t) \leq(1-\gamma \varepsilon)^{-1}\left[\left(1+C_{n} \gamma\right)\left\|\zeta_{0}\right\|+C_{X}\left(\gamma^{2} \varepsilon^{4 n-2} t^{2} e^{\varkappa_{n} \gamma \varepsilon t / 2}\right)\right],
$$

for $0 \leq t \leq T^{*}$.

Since $T=\frac{\dot{8} C_{n}}{\varkappa \varepsilon}$, if $n>2$, and if $\varepsilon$ is sufficiently small, then $T \leq T^{*}$ and we have for $0 \leq t \leq T$,

$$
Z(t) \leq(1-\gamma \varepsilon)^{-1}\left(1+\frac{3}{2} C_{n} \gamma\right) \gamma \varepsilon^{n}
$$


From the definition of $Z(t)$, this implies

$$
\|\zeta(t)\| \leq e^{-\varkappa_{n} \gamma \varepsilon t / 2}(1-\gamma \varepsilon)^{-1}\left(1+\frac{3}{2} C_{n} \gamma\right) \gamma \varepsilon^{n},
$$

or

$$
\|\zeta(T)\| \leq \gamma \varepsilon^{n}
$$

using the definition of $T$.

\section{Re-orthogonalization}

As the system evolves, the solution will remain close (at least for some time) to the cylinder of breather solutions for the $\gamma=0$ equations. However, it will drift, so that the base point on the cylinder changes with time, while the vector $\zeta$ stays orthogonal to the tangent space to the cylinder at the initial base point. In the first subsection we show that we can periodically choose new coordinates in such a way that $\zeta$ remains small for a very long time, while the frequency $\varphi$ of the base point in the cylinder changes in a controlled and computable way. The change in the base point manifests itself in the presence of the terms proportional to $\delta$ in the equation for $\zeta$. To counteract this secular growth, we will stop the evolution after a long, but finite, interval and "reset" the initial data so that the "new" initial data $\widehat{\zeta}$ is again orthogonal to the tangent space at the "new" initial point $(\widehat{\varphi}, \widehat{\vartheta})$ on the cylinder. Our approach in this section is inspired by the work of Promislow [10] on pattern formation in reaction-diffusion equations, but is complicated by the very weak dissipative properties of the semigroup $e^{t \mathcal{L}_{\varphi, \gamma}}$. In particular, we will not be able to show that the normal component, $\zeta$ of the solution is strongly contracted, but we will prove that it remains small for a very long period, during which the solution evolves close to the cylinder of breathers.

Key to this approach is the fact that in a sufficiently small neighborhood of the cylinder of breathers, the angle and phase of the point on the cylinder and the normal direction at that point provide a smooth coordinate system. More precisely, one has:

Proposition 9.1. Fix $0<\Phi_{0} \ll 1$. There exists $\mu>0$ such that for any $\bar{\varphi} \in[1-$ $\left.\Phi_{0}, 1+\Phi_{0}\right], \bar{\vartheta} \in[0,2 \pi),\|\bar{\zeta}\|<\mu$, there exists $(\widehat{\varphi}, \widehat{\vartheta}, \widehat{\zeta})$ such that

$$
e^{\mathbf{i} \bar{\vartheta}} p(\bar{\varphi})+\bar{\zeta}=e^{\mathbf{i} \widehat{\vartheta}} p(\widehat{\varphi})+\widehat{\zeta},
$$

and $\widehat{\zeta}$ is normal to the tangent space of the family of breathers at $(\widehat{\varphi}, \widehat{\vartheta})$.

Remark 9.2. The utility of this proposition is that if we choose any point near our family of breathers, we can find $(\widehat{\varphi}, \widehat{\vartheta}, \widehat{\zeta})$ to use as initial conditions for our modulation equations (7.16) with $\widehat{\zeta} \in \operatorname{Range}\left(\mathbb{P}_{\widehat{\varphi}}\right)$.

Proof. The proof is an application of the implicit function theorem. Begin by rescaling $\bar{\zeta} \rightarrow \mu \bar{\zeta}$, with $\|\bar{\zeta}\|=1$. Then we have $\widehat{\zeta}=e^{\mathbf{i} \bar{\vartheta}} p(\bar{\varphi})+\mu \bar{\zeta}-e^{\mathbf{i} \widehat{\vartheta}} p(\widehat{\varphi})$. We wish to choose $(\widehat{\varphi}, \widehat{\vartheta})$ so that $\widehat{\zeta}$ is orthogonal to the tangent space at $(\widehat{\varphi}, \widehat{\vartheta})$. Thus, we define

$$
F(\widehat{\varphi}, \widehat{\vartheta} ; \mu)=\left(\begin{array}{c}
\left\langle n_{\widehat{\varphi}, \widehat{\vartheta}}^{(1)} \mid \widehat{\zeta}\right\rangle \\
\left\langle n_{\widehat{\varphi}, \widehat{\vartheta}}^{(2)} \mid \widehat{\zeta}\right\rangle
\end{array}\right)=\left(\begin{array}{c}
\left\langle n_{\widehat{\varphi}, \widehat{\vartheta}}^{(1)} \mid\left(e^{\mathbf{i} \bar{\vartheta}} p(\bar{\varphi})+\mu \bar{\zeta}-e^{\mathbf{i} \widehat{\vartheta}} p(\widehat{\varphi})\right)\right\rangle \\
\left\langle n_{\widehat{\varphi}, \widehat{\vartheta}}^{(2)} \mid\left(e^{\mathbf{i} \bar{\vartheta}} p(\bar{\varphi})+\mu \bar{\zeta}-e^{\mathbf{i} \widehat{\vartheta}} p(\widehat{\varphi})\right)\right\rangle
\end{array}\right),
$$

and the theorem follows by finding zeros of this function. 
Note that $F(\bar{\varphi}, \bar{\vartheta} ; 0)=0$. To compute the derivative of $F$ with respect to $(\widehat{\varphi}, \widehat{\vartheta})$ we recall from the previous sections that the derivatives of $e^{\mathbf{i} \vartheta} p(\varphi)$ with respect to $\varphi$ and $\vartheta$ give precisely the two vectors $v_{\varphi, \vartheta}^{(j)}(j=1,2)$ which span the zero eigenspace. Thus, by the normalization of the vectors $n_{\widehat{\varphi}, \widehat{\vartheta}}^{(j)}$, we see that

$$
\left.D_{\varphi, \vartheta} F\right|_{\mu=0}=\left(\begin{array}{ll}
1 & 0 \\
0 & 1
\end{array}\right) .
$$

Thus, the implicit function theorem implies that there exists $\mu_{0}>0$ such that for any $|\mu|<\mu_{0}$, we have a solution $F(\widehat{\varphi}, \widehat{\vartheta} ; \mu)=0$.

Remark 9.3. Note that the size of the neighborhood $\mu_{0}$ on which we have a solution is independent of the base point $(\varphi, \vartheta)$ - thus we have good coordinates on a uniform neighborhood of our original family of breathers.

Remark 9.4. Note that the constructive nature of the proof of the implicit function theorem also results in good estimates of the size of the solutions of the equation. In particular, for small $\mu$, there exists a constant $C>0$ such that the change in the angle and phase can be estimated as:

$$
|\bar{\varphi}-\widehat{\varphi}|+|\bar{\vartheta}-\widehat{\vartheta}| \leq C \mu\left(\left|\left\langle n_{\bar{\varphi}, \bar{\vartheta}}^{(1)} \mid \bar{\zeta}\right\rangle\right|+\left|\left\langle n_{\bar{\varphi}, \bar{\vartheta}}^{(2)} \mid \bar{\zeta}\right\rangle\right|\right) .
$$

9.1. The intuitive picture. Suppose that we start from a point near our family of breathers, with coordinates $\left(\varphi_{0}, \vartheta_{0}, \zeta_{0}\right)$, with $\zeta_{0} \in \operatorname{Range}\left(\mathbb{P}_{\varphi_{0}}\right)$. We allow the system to evolve for a time $T$ to be specified below. After this time, we will have reached a point $\left(\varphi_{1}=\right.$ $\left.\varphi(T), \vartheta_{1}=\vartheta(T), \zeta_{1}=\zeta(T)\right)$. In terms of our original variables, this point will be

$$
w_{1}=e^{\mathbf{i}\left(\varphi_{1} T+\vartheta_{1}\right)}\left(p\left(\varphi_{1}\right)+z_{1}\right),
$$

where $z_{1}=\left(\xi_{1}+\mathbf{i} \eta_{1}\right)$, with $\left(\xi_{1}, \eta_{1}\right)^{\top}=\zeta_{1}$. The point is that $\zeta_{1}$ is no longer orthogonal to the tangent space to the cylinder of breathers at the point $\left(\varphi_{1}, \vartheta_{1}\right)$. This leads to secular growth in $\zeta$, and eventually, we would loose control of this evolution. To prevent this, we re-express the point $w_{1}$ in terms of new variables $(\widehat{\varphi}, \widehat{\vartheta}, \widehat{\zeta})$, with $\widehat{\zeta}$ orthogonal to the tangent space at $(\widehat{\varphi}, \widehat{\vartheta})$, and restart the evolution of (7.16) with these new initial conditions. The only complication is that we must keep careful track of how much we change the various variables in the course of this re-orthogonalization process. We now explain how this is done.

Without loss of generality assume that we have chosen the "stopping time" $T$ so that the phase $e^{\mathbf{i}\left(\varphi_{1} T+\vartheta_{1}\right)}=1$. (If this is not the case, we can always use the phase invariance of the equation to rotate the solution so that this does hold.) Then, after time $T$, the trajectory of our system will have reached the point

$$
w_{1}=p\left(\varphi_{1}\right)+z_{1} .
$$

By Proposition 9.1 we know that there exists $(\widehat{\varphi}, \widehat{\vartheta}, \widehat{\zeta})$ with

$$
w_{1}=p\left(\varphi_{1}\right)+z_{1}=e^{\mathbf{i} \widehat{\vartheta}} p(\widehat{\varphi})+\widehat{\zeta}
$$

and $\widehat{\zeta}$ is normal to the cylinder of breathers at $(\widehat{\varphi}, \widehat{\vartheta})$. We now restart the evolution of the modulation equations (7.16) and follow the evolution as before. 
The last thing we need to control the long-time evolution of the system is to estimate by how much we change $\varphi$ and $\zeta$ in the course of this re-orthogonalization. (The change in $\vartheta$ is inconsequential since it does not affect the magnitude of the solution, and since the phase-invariance of the equations of motion allows to always rotate the system back to zero phase if needed.) The change from $\varphi_{1}$ to $\widehat{\varphi}$ is estimated with the aid of the implicit function theorem.

We know that the vectors $\left\langle n_{\varphi, \vartheta}^{(j)}\right|$ depend smoothly on $\varphi$ and hence

$$
\begin{aligned}
\mid\left\langle n_{\varphi_{1}}^{(1)} \mid \zeta_{1}\right\rangle & \leq\left|\left\langle n_{\varphi_{1}}^{(1)} \mid \zeta_{1}\right\rangle-\left\langle n_{\varphi_{0}}^{(1)} \mid \zeta_{1}\right\rangle\right|+\left|\left\langle n_{\varphi_{0}}^{(1)} \mid \zeta_{1}\right\rangle\right| \\
& \leq\left|\left\langle n_{\varphi_{1}}^{(1)} \mid \zeta_{1}\right\rangle-\left\langle n_{\varphi_{0}}^{(1)} \mid \zeta_{1}\right\rangle\right| \leq C \delta(T)\left\|\zeta_{1}\right\| \leq C \delta(T) \gamma \varepsilon^{n} .
\end{aligned}
$$

Here, the first inequality just uses the triangle inequality, the second the fact that $\zeta_{1}$ is orthogonal to $n_{\varphi_{0}}^{(1)}$ by construction, the third uses Cauchy-Schwarz, plus the smooth dependence of the normal vectors on $\varphi$, and the last, the estimate on $\zeta_{1}$ coming from Theorem 8.2. If we combine this estimate with (9.1), we see that the change in $\varphi$ from $\varphi_{1}$ to $\widehat{\varphi}$ produced by the re-orthogonalization is extremely small.

It remains to estimate the corresponding change in $\zeta$ when we replace $\zeta_{1}$ by $\widehat{\zeta}$. We have

$$
p\left(\varphi_{1}\right)+z_{1}=e^{\hat{i \vartheta}} p(\widehat{\varphi})+\widehat{z},
$$

where as usual $\widehat{z}=\widehat{\xi}+\mathbf{i} \widehat{\eta}$, with $\widehat{\zeta}=(\widehat{\xi}, \widehat{\eta})^{\top}$. Again, using the fact that $p(\varphi)$ depends smoothly on $\varphi$, plus estimates on the difference in $\varphi_{1}$ and $\widehat{\varphi}$ given by (9.2) and similar estimates for the $\widehat{\vartheta}$, we see that

$$
\left\|\zeta_{1}-\widehat{\zeta}\right\| \leq C_{R} \delta(T) \gamma \varepsilon^{n}
$$

or

$$
\|\widehat{\zeta}\| \leq\left(1+C_{R} \delta(T) \gamma \varepsilon^{n}\right)\left\|\zeta_{1}\right\|
$$

for some finite $R_{2}$.

\section{Iterating}

The estimates of the previous section show that if we take initial conditions for (1.3) close to the cylinder of breathers for the undamped equations, and if we express that initial point as

$$
w_{0}=p\left(\varphi_{0}\right)+z_{0},
$$

with $\zeta_{0}=\left(\Re\left(z_{0}\right), \Im\left(z_{0}\right)\right)^{\top} \in \operatorname{Range}\left(\mathbb{P}_{0}\right)$ and $\left\|\zeta_{0}\right\| \leq \gamma \varepsilon^{n}$, then $\varphi, \vartheta$, and $\zeta$ will evolve via (8.2)-(8.4) and after a time $T=\frac{4 C_{n}}{\varkappa_{n} \varepsilon}$ we will have

$$
\begin{aligned}
\varphi(T)-\varphi_{0} & =-2 \gamma \varepsilon^{2 n-1} T\left(1+\mathcal{O}\left(\varepsilon^{1 / 2}\right)\right), \\
\|\zeta(T)\| & \leq(1-\gamma \varepsilon)^{-1}\left(1+\frac{3}{2} C_{n} \gamma\right) \varepsilon^{n} .
\end{aligned}
$$

As usual we ignore the evolution of $\vartheta$ since any $\vartheta$ dependence of the solution can be removed using the phase invariance of the problem.

As discussed in Sect. 9, $\zeta(T)$ will not lie in $\operatorname{Range}\left(\mathbb{P}_{\varphi(T)}\right)$. Thus, we now reorthogonalize. To see what is involved, consider again Fig. 2. 


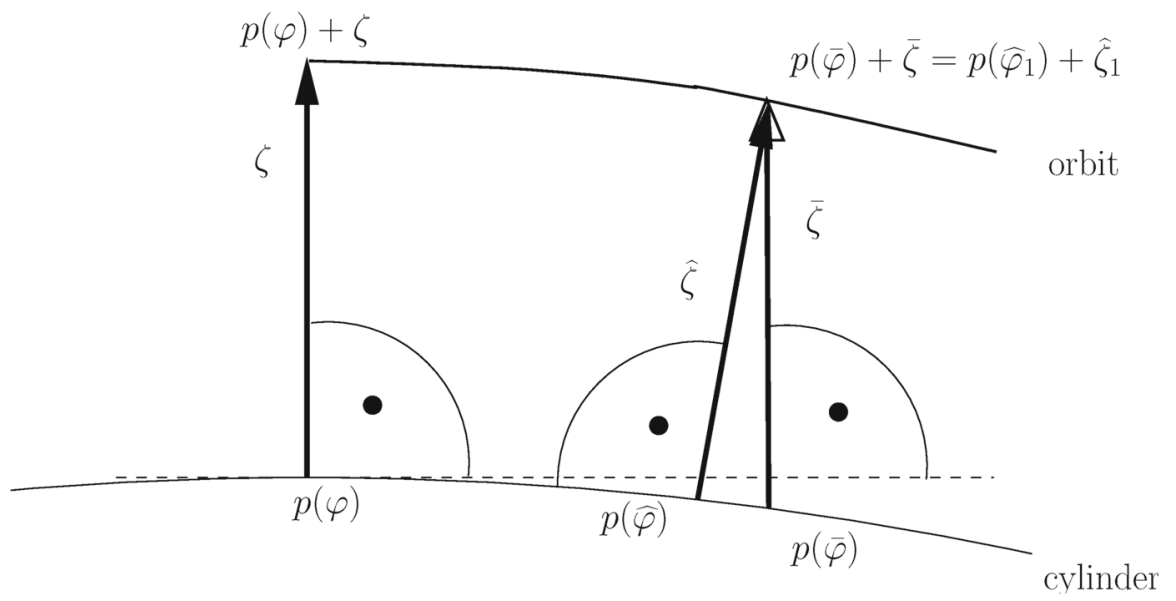

Fig. 2. Illustration of the re-orthogonalization process. At time 0 , the orbit starts at a distance $\|\zeta\|$ from the base point $p(\varphi)$, which lies on the cylinder (shown as a line). $\zeta$ is orthogonal to the tangent at the point $p(\varphi)$ on the cylinder (this is the 2-dimensional subspace of 0 eigenvalues). At time $T$, the solution has moved to $p(\bar{\varphi})+\bar{\zeta}$, with $\bar{\zeta}$ still orthogonal to the tangent space at $p(\varphi)$. The re-orthogonalization consists of finding a new base point $\widehat{\varphi}$ in such a way that $p(\bar{\varphi})+\bar{\zeta}=p(\widehat{\varphi})+\widehat{\zeta}$ and $\widehat{\zeta}$ is orthogonal to the tangent space at $p(\widehat{\varphi})$. This solution is found by the implicit function theorem. Note that $\|\widehat{\zeta}\|$ might be larger than $\|\zeta\|$, but this is compensated by the contraction induced by semigroup due to the dissipation

This means we reexpress

$$
w(T)=e^{\mathbf{i} \varphi(T) T} p(\varphi(T))+z(T)=e^{\mathbf{i}(\widehat{\varphi} T+\widehat{\vartheta})} p(\widehat{\varphi})+\widehat{z},
$$

where as usual, $\widehat{z}=(\widehat{\xi}+\mathbf{i} \widehat{\eta})$, with $(\widehat{\xi}, \widehat{\eta})=\widehat{\zeta}$ and $\widehat{\zeta} \in \operatorname{Range}\left(\mathbb{P}_{\widehat{\varphi}}\right)$.

We now recall the estimates for the change in $\varphi$ and $\zeta$ produced by the re-orthogonalization. First, from (9.2), plus the estimate on $\delta(T)$ from Lemma 8.6, we have

$$
|\varphi(T)-\widehat{\varphi}| \leq C \delta(T) \varepsilon^{n},
$$

and hence by the triangle inequality we see that

$$
\left|\left(\varphi_{0}-\widehat{\varphi}\right)+2 \gamma \varepsilon^{2 n-1} T\right| \leq 4 \gamma \varepsilon^{2 n-1 / 2} T,
$$

i.e., to leading order $\varphi_{0}-\widehat{\varphi} \approx \varphi_{0}-\varphi(T)$.

Likewise, from (9.3), we have

$$
\begin{aligned}
\|\widehat{\zeta}\| & \leq\|\zeta(T)\|+\|\zeta(T)-\widehat{\zeta}\| \\
& \leq e^{-x_{n} \gamma \varepsilon T / 2}(1-\gamma \varepsilon)^{-1}\left(1+\frac{3}{2} C_{n} \gamma\right) \varepsilon^{n}+4 \gamma \varepsilon^{2 n-1} T \\
& \leq \gamma \varepsilon^{n}
\end{aligned}
$$

for $\varepsilon$ sufficiently small. If we look at the second line above, we see how the contraction, and the "waiting" for a time $T$ come in: Namely, the first factor contracts, because of the estimates on the semigroup (and the dissipation), while the next two factors come from the reprojection and the prefactor from the bound on the semigroup. 
Thus, we can begin to evolve our equation of motion starting from the point $w(T)$, but now expressed as

$$
w(T)=e^{\mathbf{i}(T \widehat{\varphi}+\widehat{\vartheta})} p(\widehat{\varphi})+\widehat{\zeta},
$$

where $\widehat{\zeta} \in \operatorname{Range}\left(\mathbb{P}_{\widehat{\varphi}}\right)$, and $\|\widehat{\zeta}\| \leq \gamma \varepsilon^{n}$. Thus, the new representation for $w(T)$ has the same properties as the representation of $w_{0}$ that we started with, and hence we can continue to evolve our trajectory which will remain close to the cylinder of breathers.

\section{Conclusions and Future Directions}

We have proven that the presence of breather solutions leads to very slow energy decay in discrete nonlinear Schrödinger equations. There are many other types of lattice dynamical systems that possess breather solutions such as discrete Klein-Gordon equations or Fermi-Pasta-Ulam-Tsingou models. (For a recent survey of such results see [13].) It would be interesting to see if breathers play a similar role in the transport of energy through lattices governed by such equations. In addition, it is clear that, at least intuitively, the reason for the slow energy decay induced by the breathers is related to their strong localization properties which means that most of the energy of the system is localized far from the region in which the dissipation acts. Thus it would also be interesting to investigate systems whose breathers are either more or less strongly localized than those of the NLS system studied here $[14,15]$.

Acknowledgements. We thank Noé Cuneo and Pierre Collet for useful discussions and the referee for helpful suggestions. The research of CEW was supported in part by the US NSF under Grant Number DMS-1813384.

Funding Open access funding provided by University of Geneva.

Open Access This article is licensed under a Creative Commons Attribution 4.0 International License, which permits use, sharing, adaptation, distribution and reproduction in any medium or format, as long as you give appropriate credit to the original author(s) and the source, provide a link to the Creative Commons licence, and indicate if changes were made. The images or other third party material in this article are included in the article's Creative Commons licence, unless indicated otherwise in a credit line to the material. If material is not included in the article's Creative Commons licence and your intended use is not permitted by statutory regulation or exceeds the permitted use, you will need to obtain permission directly from the copyright holder. To view a copy of this licence, visit http://creativecommons.org/licenses/by/4.0/.

Publisher's Note Springer Nature remains neutral with regard to jurisdictional claims in published maps and institutional affiliations.

\section{References}

1. Eckmann, J.P., Wayne, C.: Breathers as Metastable States for the Discrete NLS Equation. Discrete Contin. Dyn. Syst. A 38, 6091 (2018)

2. Cuneo, N., Eckmann, J.P., Wayne, C.: Energy dissipation in Hamiltonian chains of rotators. Nonlinearity 30, R81 (2017)

3. MacKay, R.S., Aubry, S.: Proof of existence of breathers for time-reversible or Hamiltonian networks of weakly coupled oscillators. Nonlinearity 7(6), 1623 (1994)

4. Flach, S., Willis, C.R.: Discrete breathers. Phys. Rep. 295(5), 181 (1998). https://doi.org/10.1016/S03701573(97)00068-9

5. Iubini, S., Lepri, S., Livi, R., Oppo, G.L., Politi, A.: A chain, a bath, a sink, and a wall. Entropy 19(9), 1 (2017). https://doi.org/10.3390/e19090445

6. Danieli, C., Campbell, D.K., Flach, S.: Intermittent many-body dynamics at equilibrium. Phys. Rev. E 95, 060202 (2017). https://doi.org/10.1103/PhysRevE.95.060202

7. Weinstein, M.: Modulational stability of ground states of nonlinear Schrödinger equations. SIAM J. Math. Anal. 16, 472 (1985). https://doi.org/10.1137/0516034 
8. Pego, R., Weinstein, M.: On asymptotic stability of solitary waves. Phys. Lett. A 162, 263 (1992)

9. Pego, R., Weinstein, M.: Asymptotic Stability of Solitary Waves. Commun. Math. Phys. 164, 305 (1994). https://doi.org/10.1007/BF02101705

10. Promislow, K.: A renormalization method for modulational stability of quasi-steady patterns in dispersive systems. SIAM J. Math. Anal. 33(6), 1455 (2002). https://doi.org/10.1137/S0036141000377547

11. Mehrmann, V., Xu, H.: Perturbation of purely imaginary eigenvalues of Hamiltonian matrices under structured perturbations. Electron. J. Linear Algebra 17, 234 (2008). https://doi.org/10.13001/1081-3810. 1261

12. Kato, T.: Perturbation Theory for Linear Operators. Springer, Berlin (1984). Second corrected printing of the second edition

13. Flach, S., Gorbach, A.V.: Discrete breathers—advances in theory and applications. Phys. Rep. 467(1), 1 (2008)

14. James, G., Starosvetsky, Y.: Breather solutions of the discrete p-Schrödinger equation. In: CarreteroGonzález, R., Cuevas-Maraver, J., Frantzeskakis, D., Karachalios, N., Kevrekidis, P., Palmero-Acebedo, F. (eds.) Localized Excitations in Nonlinear Complex Systems. Springer, Berlin (2014)

15. Flach, S.: Breathers on lattices with long range interaction. Phys. Rev. E 58, R4116 (1998). https://doi. org/10.1103/PhysRevE.58.R4116

Communicated by J. Marklof 